\title{
Risk-Sharing and Investment in Concentrated Markets*
}

\author{
Daniel Neuhann ${ }^{\dagger} \quad$ Michael Sockin
}

January 2018

\section{PRELIMINARY}

\begin{abstract}
We study investment and risk sharing in complete markets when agents internalize their impact on asset prices. Quantity shading of state-contingent claims by buyers and sellers generates excess exposure to idiosyncratic risk and low asset pledgeability. This depresses investment, the risk-free rate, and aggregate productivity. Rents from market power distort and misalign agents' marginal valuations of state-contingent returns, rendering risk-sharing constrained inefficient and as if markets were competitive but incomplete. When there is limited commitment, sellers face borrowing constraints that limit their ability to strategically restrict supply, thereby reallocating market power to buyers. When markets are decentralized, agents distort investment to capture arbitrage profits by acting as pass-through intermediaries.
\end{abstract}

${ }^{*}$ We thank Jonathan Berk, John Hatfield, Yiming Ma, Gregor Matvos, Aaron Pancost, Neng Wang, and seminar participants at UT Austin for helpful comments. We thank Mahyar Sefidgaran for excellent research assistance.

${ }^{\dagger}$ UT Austin. daniel.neuhann@mccombs.utexas.edu

${ }_{\ddagger}^{\ddagger}$ UT Austin. michael.sockin@mccombs.utexas.edu 


\section{Introduction}

The key roles of a financial system are to facilitate the sharing of risks and to allocate capital to its most productive uses. In competitive financial markets, market prices serve to efficiently coordinate much of this activity. Yet there is growing evidence that many modern financial systems are far from perfectly competitive. The Office of the Comptroller of the Currency, for instance, estimates that over $90 \%$ of the notional amount of interest-rate swaps is accounted for by four banks, while over $95 \%$ of the CDS market is accounted for by only three banks. 1 Corbae and Levine (2018) shows that the five largest U.S. banks held $47 \%$ of total U.S. bank assets in 2015. In the U.K., France, Germany, Italy, and Canada, the same number ranges from $71 \%$ to $84 \%$; in China it is $53 \%$. De Loecker, Eeckout, and Unger (2018) estimate that markup in the financial industry have increased alongside those in other industries since the 1980s. Hau, Hoffmann, Langfield, and Timmer (2017) document substantial non-competitive pricing in over-the-counter derivatives markets.

The aggregate and distributional implications of this concentration are still not wellunderstood, however. To analyze these issues, we provide a theoretical framework to study the impact of imperfect competition in financial markets on productive efficiency, risksharing, and welfare. We study an economy in which a finite number of large agents with production opportunities can trade Arrow-Debreu securities subject to two strategic constraints. First, large agents internalize the impact of their trades on market prices, and exercise market power. Second, similar to Alvarez and Jermann (2000), agents have limited commitment to repay their obligations. We show that market power alone is sufficient to hamper risk-sharing, depress investment, reduce the risk-free rate, and lower aggregate productivity. Market power also interacts with limited commitment to further amplify these effects. Our results are consistent with recent U.S. evidence of the increasing concentration in financial markets and the secular declines in investment, risk-free rates, and productivity (e.g. Gutierrez and Philippon (2016)). We also show that, when markets are segmented, market power can lead to pure arbitrage profits.

Our model features a finite number of strategic agents, each endowed with a production technology exposed to both aggregate and agent-specific risk. These technologies may represent direct investments in real assets or lending opportunities to unmodeled borrowers in the real economy. Agents can trade a complete set of Arrow-Debreu securities to finance

\footnotetext{
${ }^{1}$ See the OCC's Quarterly Report on Bank Trading and Derivatives Activities, 2017 Q4.
} 
investment and share risks, and they internalize their portfolio choices affect asset prices through Cournot competition ${ }^{2}$ To exploit market power, agents strategically shade down quantities to tilt asset prices in their favor. For buyers of assets, the strategic behavior of sellers raises the costs of obtaining insurance against their project-specific risks. For sellers, the strategic behavior of buyers lowers the price at which they can sell claims on future cash flows, reducing the pledgeability of investment. The first effect deters risky investments and lowers the risk-free rate, while the second depresses volumes. Market power, however, also has a rich interaction with other financial frictions. When agents face endogenous borrowing constraints, sellers have weaker incentives to shade down demand beyond the level implied by the constraint. Limited commitment, consequently, reallocates de-facto market power to buyers, amplifying their distortion to investment and asset prices at the expense of sellers.

Whether an agent is a buyer or seller in a given security market is jointly determined by the exogenous risk profile of its production technology and its endogenous investment choices. This is because an agent sells a security when his production returns are correlated with the security's payoffs, and is a buyer otherwise. The sorting of agents across markets, and thus market power and asset prices, are therefore fundamentally linked to the distribution of production technologies across agents. Accordingly, risk premia, investment levels and productive efficiency are not invariant to changes in this productivity distribution even if the aggregate technology frontier is unchanged. Moreover, agents may strategically distort investment toward low-return technologies in order to earn market power rents, and to increase the pledgeability of their balance sheets.

The distribution of production returns also affects the market shares of agents in each security. When the returns to production are highly dispersed, there are fewer natural sellers of any given security than potential buyers. As a result, the motives of sellers to distort prices are typically stronger than those of buyers in our economy. Despite the fact that there are strategic agents on both sides of the market, market power increases rather than decreases asset prices, in equilibrium.

We provide a number of additional characterization results. First, asset prices can be decomposed into three components: a standard risk premium, a term reflecting binding borrowing constraints, similar to Alvarez and Jermann (2000), and a term linked to market

\footnotetext{
${ }^{2}$ To maintain tractability for examples, and ensure uniqueness of price impact, we assume that a nonstrategic competitive fringe participates in every asset market. Our main qualitative insights, however, do not rely on this assumption, and we impose only mild regularity conditions on the fringe's demand schedule.
} 
power. The last term implies the existence of arbitrage profits, which are absent in a competitive setting. Second, risk-sharing arrangements in our setting with complete markets and market power appear as if markets are competitive, but incomplete. Specifically, a set of securities with a strictly smaller asset span is sufficient to replicate the observed degree of risk-sharing among agents given any investment policy.

Our results highlight a distinction between what we will refer to as perfect, imperfect, and incomplete risk-sharing. Perfect risk-sharing is when marginal rates of substitutions (MRS) are aligned state by state across agents. Imperfect risk-sharing is a weaker notion requiring that state prices, which are the state-specific sums of marginal rates of substitution and Lagrange multipliers on binding portfolio constraints, are aligned even if MRSs are not. Finally, risk-sharing is incomplete if both both MRSs and state prices are dispersed.

It is well-known that risk-sharing is perfect in frictionless, complete markets, and imperfect in complete markets when agents face position limits, such as in models of limited commitment. In our model of complete markets with market power, instead, risk-sharing is incomplete. This is because, contrary models of limited commitment, agents voluntarily under-provide insurance in order to capture price rents, rather than being forced to limit risk-sharing by position limits. Sellers thus remain exposed to states in which their output is high in excess of what potential borrowing limits might imply, while buyers garner too little insurance against states in which their output is low, even though their asset purchases are not constrained. It follows that the consumption allocation generated security trading in our model is generically constrained inefficient, even absent borrowing limits. This highlights potential similarities between our model of market power and the risk-sharing implications of alternative settings in which frictions, such as asymmetric information stemming from adverse selection or moral hazard, generate rents that prevent the alignment of state prices. Constraints that do not generate rents, such as borrowing, position, and limited commitment constraints, thus force imperfect risk-sharing upon agents, while frictions that induce rents introduce voluntary wedges that lead to incomplete risk-sharing.

Since investment is endogenous in our model, incomplete risk-sharing can also hamper productive efficiency by discouraging investment in high-return, high-idiosyncratic risk technologies. This mechanism also generates spillovers from market power across multiple securities: if agents respond by distorting investment, the underprovision of insurance in one market may lower the availability of funds in other states of the world, further reducing 
risk-sharing. These effects are amplified when borrowing constraints bind.

Finally, we consider an extension in which agents can only trade with a fixed set of counterparties, as in an incomplete network. Asset prices now provide information about the underlying market structure. Not only can the same security trade at different prices across the network, but risk premia are lower for the debt of agents who act as pass-through financiers, and double-margin across segmented markets. When links must be formed at a cost, these arbitrage profits from double-margining can provide incentives for an agent to establish such connections with trading partners, forming a core-periphery configuration.

\section{$1.1 \quad$ Related Literature}

Our paper is most closely related to the literature on asset pricing and efficiency in models of limited commitment, including Alvarez and Jermann (2000), Kehoe and Levine (1993) and Kocherlakota (1996). We differ from these studies in that we study strategic agents with market power who trade claims on risky production, rather than endowments. With regard to intermediaries, Rochet and Tirole (1996) argues that interbank liabilities provide incentives for cross-monitoring, while Leitner (2005) shows that contagion risk stemming from investment complementarities can foster cooperation in the absence of commitment. We abstract from moral hazard issues in monitoring and from financial contagion to instead focus on the feedback and strategic complementarities between state-continent risk-sharing arrangements and production decisions.

A large literature, including Allen and Gale (2000), Eisenberg and Noe (2001), Elliott, Golub, and Jackson (2014), Acemoglu, Ozdaglar, and Tahbaz-Salehi (2015), and Erol (2018), examines the incentives for intermediaries to share risk and the implications of interbank markets for default, contagion, and crises. Denbee, Julliard, Li, and Yuan (2018) assesses the value of liquidity in interbank markets during the financial crisis, while Barattieri, Moretti, and Quadrini (2017) investigates the relevance of network effects in explaining bank leverage during this time period. Closest to our study is Zawadowski (2013), who shows that it may be privately optimal but socially inefficient to not obtain insurance against certain insurable risks. A common theme in these papers is that markets are incomplete, and production is either absent or highly stylized. By allowing for complete markets, agents in our model can achieve state-contingent capital flows without resorting to inefficient default. In addition, our asset pricing predictions do not depend on a particular market structure, as with incomplete 
markets, and our implications for real distortions arise from the ex ante misallocation of capital, rather than from inefficient early liquidation.

Our work is also related to the literature studying strategic behavior in financial markets under symmetric information, both with complete and incomplete markets $\mathrm{S}^{3}$ Donaldson (1992) explores how banks can strategically deploy liquidity at non-competitive rates. Basak (1997) assesses the asset pricing implications of the presence of a non-competitive investor in an endowment economy. Pritsker (2005) considers large traders with price impact who can front-run distressed sales. Weretka (2011), Carvajal and Weretka (2012) and Rostek and Weretka (2015) investigate the role of constant price impact for risk-sharing in thin financial markets. Koijen and Yogo (forthcoming) examines the vulnerability of financial markets to contagion among institutional investors who have price impact when trading in asset markets. Glebkin (2017) and Malamud and Teguia (2017) explore nonlinear, symmetric equilibria with large traders and non-normal payoffs. A key difference in our setting is that we investigate how strategic trading interacts with production and consider asymmetric equilibria. In addition, our choice of market structure can be understood as a form of financial innovation, similar to the model of endowment risk sales of Carvajal, Rostek, and Weretka (2012).

\section{Model}

\section{$2.1 \quad$ Environment}

Consider two dates $t=\{1,2\}$ and $N$ agents, indexed by $i \in\{1, \ldots, N\}$. For our main application, these agents are intermediaries that can be thought of broadly as including banks, bank holding companies, and other intermediaries such as asset managers and money market mutual funds. Our framework, however, can also be more applied to study the strategic interaction between firms, where leverage constraints in the sequel can be reinterpreted as capacity constraints. ${ }^{4}$

\footnotetext{
${ }^{3}$ There is also a vast literature studying strategic trading under asymmetric information, including Kyle (1985), Kyle (1989), Bhattacharya and Spiegel (1991), Foster and Viswanathan (1996), Vayanos (1999), Vayanos (2001), Anthropelos (2017), Rostek and Weretka (2012), and Rostek and Weretka (2015).

${ }^{4}$ With this interpretation, the output in different states can alternatively be viewed as reflecting differentiated goods, $z \in \mathcal{Z}$, over which each agent needs to produce a final output good according to the nonlinear production technology, $\sum_{z \in \mathcal{Z}} \pi(z) u\left(c_{i 2}(z)\right)$. With this interpretation, firm $i$ 's technology $y_{i}$ produces some subset of these intermediate goods.
} 
Agent $i$ is endowed with initial equity $e_{i}>0$ at $t=1$. This initial equity reflects funding from end clients, such as depositors and institutional investors. As in practice, this initial capital is slow-moving and does not respond to agents' investment opportunities. All agents have access to an investment opportunity and a storage technology that can transfer resources $s_{i} \geq 0$ elastically across dates 1 and 2 at a riskless rate of transformation $R_{f} \geq 1$. Storage cannot be negative to ensure that, if an agent wants to borrow additional resources, it must do so in a wholesale lending market from other agents, and will often at an implicit interest rate that differs from $R_{f}$.

At $t=1$, agent $i$ has access to an investment opportunity that can transform $k_{i}$ units of capital into $y_{i} k_{i}$ units of a consumption good at $t=2$, where $y_{i}$ is the productivity of agent $i$ realized at time 2 . These investment opportunities can be thought of as portfolios of projects in which agents can invest, and differences in productivity as differences in the firms in the loan portfolios, their risk profile, and the markets in which they participate. Capital is in elastic supply, and can be produced at an unit marginal cost.

We assume that the productivity of agent $i y_{i}$ is bounded a.s., and can be expressed as $y_{i}=Y+\varepsilon_{i} \geq 0$, where $Y$ is correlated risk, and $\varepsilon_{i}$ is risk specific to agent $i$. We index states of the world by $z \in \mathcal{Z}$, equipped with its natural tribe and a probability measure $\pi(z)$, which includes all possible realizations of agents' productivities. Since there are a finite number of agents, total production in the economy $y=\sum_{i=1}^{N} y_{i}$ is a random variable. In what follows, we use vector notation, in which case $\vec{x} \geq \vec{y}$ implies $x_{i} \geq y_{i}$ for all $i$, while $\vec{x}>\vec{y}$ implies $x_{i} \geq y_{i}$ for all $i$ with at least one inequality sharp. In addition, $\vec{x}_{-i}$ is the vector $\vec{x}_{i}$ omitting the $i^{\text {th }}$ element.

Agents can trade claims to future output with each other at $t=1$ in centralized Walrasian markets $5^{5}$ These can be thought of as trade credit extended for future deliverables, or as financial claims that pay at $t=2$. Markets are complete in that agents can potentially contract on output at $t=1$ contingent on any state of the economy that could realize at date $t=2$. A contract issued by agent $i$ is that pays off a unit of the numeraire good in state $z \in \mathcal{Z}$ at $t=2$ has price $q(i, z)$ at $t=1.6$ Similar to Alvarez and Jermann (2000), however, there is limited commitment in that an agent can default on its obligations if it does not

\footnotetext{
${ }^{5}$ Although our market structure precludes bilateral contracting between agents, this is not essential for our qualitative insights. Our results would remain true even in a duopoly version of our model, and as long as any bilateral contracting is publicly observable and cannot sidestep the limited commitment friction.

${ }^{6}$ As there is no long-term relationships among agents in this static setting, it is without loss not to index securities by issuer / creditor pairs.
} 
find it optimal to deliver its goods at $t=2$. The lack of pledgeability captures legal issues in enforcement of contracts. Agents cannot partially default and default is costly in that it entails a deadweight loss. Specifically, a fraction $\xi$ of an agent's final cash flows are lost at $t=2$ upon default. Although all contingent claims are non-recourse, distress costs provide a tradeoff against default.

Since each agent exercises market power, it will profit from trading two securities that reference the same state $z \in \mathcal{Z}$. Given that exchanging securities is a zero-sum trade, both agents cannot profit from it and, as such, they will not exchange cross-holdings.7 Each agent will only take one side of a given security market, indexed by issuer and state.

The budget constraint of agent $i$ at $t=0$ is then:

$$
c_{1 i}+k_{i}+\sum_{z \in \mathcal{Z}}\left(\vec{a}_{i}(z)\right) \vec{q}(z)+s_{i} \leq e_{i}-\sum_{z \in \mathcal{Z}} q(i, z) a_{i}(i, z),
$$

where $c_{0 i}$ is initial consumption, and we have allowed free disposal of resources. Purchases of capital $k_{i}$ and claims to state $z, \vec{a}_{-i}(z)$, from other agents, and storage $s_{i}$ must be financed from initial equity and whatever claims to output agent $i$ issues across states $z, a_{i}(i, z)$, at a price $q(i, z)$. As a result of the structure of the network and market power, it is not sufficient to keep track of one state price per state. Since agents who are not connected directly cannot attempt to align their marginal utilities across states, they need not agree on state prices. Furthermore, since agents internalize their price impact, they have incentive to spread their demand for contingent claims within a state across many issuers to minimize the cost of insurance, and consequently we cannot impose one market-clearing condition and one market-clearing price for claims to output referencing each state of nature.

If agent $i$ delivers on its obligations at $t=2$, it receives final consumption of $c_{i 2}^{N D}(z)$ :

$$
c_{i 2}^{N D}(z)=y_{i}(z) k_{i}+\sum_{j=1}^{N} a_{i}(j, z)+R_{f} s_{i}
$$

while if it defaults, it receives $c_{i 2}^{D}(z)$ :

$$
c_{i 2}^{D}(z)=(1-\xi)\left(y_{i}(z) k_{i}+\sum_{j \neq i}^{N} a_{i}(j, z)+R_{f} s_{i}\right) .
$$

\footnotetext{
${ }^{7}$ In this sense, cross-holdings arise in equilibrium only if markets are incomplete. See Donaldson and Piacentino (2017) for a recent variation on this argument.
} 
In contrast to Kehoe and Levine (1993), in which private production would serve only to tighten the individual rationality constraints to repay in every state, production in our setting relaxes these constraints since it increases the loss in consumption agent $i$ would suffer if it defaulted.

Since markets are complete, we can focus our attention on allocations in which agents do not default in equilibrium. As agents are rational, the price of any security referencing a default state for an issuer commands zero price, providing no initial benefit ex ante to the seller in the trading game, and default further entails a deadweight loss if the state realizes 8 Furthermore, since agents are risk-averse, and their budget constraints are linear, introducing additional randomization states on which to contract without partial default would only reduce the aggregate value of state contingent claims tied to each output state by introducing nonfundamental volatility into each agent's stochastic discount factor.

Agents are risk-averse and have cardinal utility index $u_{t}(z)$ over initial consumption, $c_{i 1}$, and their expected final consumption, $c_{i 2}(z) \in\left\{c_{i 2}^{N D}(z), c_{i 2}^{D}(z)\right\}$, where $u_{2}(\cdot)$ is strictly increasing, continuously differentiable, satisfies the Inada condition, and $u_{2}^{\prime}(\cdot)$ is homothetic of degree $\gamma>0$ and convex. In the sequel, unless specified otherwise, $u_{1}(\cdot)$ satisfies these properties as well. Agent $i$ chooses its policies to maximize its ex ante expected utility:

$$
\begin{aligned}
U_{1}^{i}\left(e_{i}, \vec{e}_{-i}\right) & =\sup _{\left\{c_{i 1}, k_{i}, s_{i}, \vec{a}_{i}(z)\right\}} u_{1}\left(c_{i 1}\right)+\sum_{z \in \mathcal{Z}} \pi(z) u_{2}\left(c_{i 2}^{N D}(z)\right), \\
\text { s.t. } & : \quad u_{2}\left(c_{i 2}^{N D}(z)\right) \geq u_{2}\left(c_{i 2}^{D}(z)\right) \forall z \in \mathcal{Z}
\end{aligned}
$$

where portfolio actions are taken at $t=1$.

As is standard practice with models of strategic interaction in concentrated markets, we assume that there is a competitive fringe of outside fundamental investors with deep pockets $9^{9}$ To cater this notion to our complete markets setting, we assume each type of outside investor invests in only one security, referenced by an issuer $i$ and state $z$. This not only makes transparent our mechanism of how agents' risk-sharing with price impact shapes the formation of agent networks and their stability, but also prevents the outside investors

\footnotetext{
${ }^{8}$ To have equilibria multiplicity, agents effectively must have identical state contingent claims across two randomization states that have the same production outcomes. Agents that differ in their repayment behavior across the two auxiliary states would only be able to issue claims against the state in which they do not default, leading them to (weakly) prefer not to have the randomization state.

${ }^{9}$ Such a competitive fringe, which are the residual claimant to the securities of agents, are also present in Eisenberg and Noe (2001), Xiong (2001), Brunnermeier and Pedersen (2005), Pritsker (2005), and Koijen and Yogo (forthcoming).
} 
from trivially unraveling the network effects in our model, since they would arbitrage price differences across markets if they could trade competitively in all securities ${ }^{10}$ We collectively refer to this fringe as agent $N+1$. This fringe can be viewed, for instance, as a continuum of specialized small banks with limited risk-absorbing capacity possibly arising from balancesheet or risk-management constraints.

We assume that outside investors in claims issued by agent $i$ referencing state $z$ have initial resources $e_{0}$, and collectively choose their demand to maximize their utility over final consumption:

$$
w_{0}=\sup _{D(i, z)} E\left[F\left(r \frac{e_{0}}{N|\mathcal{Z}|}+\left(\mathbf{1}_{\left\{z^{\prime}=z\right\}}-r q(i, z)\right) D(i, z), z\right)\right],
$$

and we assume they have access to unrestricted access to riskfree borrowing and lending at the interest rate $r \geq 1$. That they can borrow and lend freely reflects that they have deep pockets. The optimization program gives rise to the FONC:

$$
\frac{\pi(z)}{1-\pi(z)} \frac{F^{\prime}\left(r \frac{e_{0}}{N|\mathcal{Z}|}+(1-r q(i, z)) D(i, z), z\right)}{F^{\prime}\left(r \frac{e_{0}}{N|\mathcal{Z}|}-r q(i, z) D(i, z), z\right)} \frac{1-r q(i, z)}{r q(i, z)}=1
$$

We assume we can express their demand, $D(i, z)$, if agent $i$ issues the claim:

$$
D(i, z)=f_{i}(q(i, z), z) \sum_{j=1}^{N} e_{j}
$$

where $f_{i}(\cdot, z)$ is a strictly decreasing $\mathcal{C}^{2}$ function of its first argument, and $q(i, z) f_{i}(\cdot, z)$ is bounded from above $\left.\forall(i, z) 1^{11}\right|^{2}$ That $f_{i}(\cdot, z)$ is strictly decreasing in its first argument reflects that state contingent claims are normal goods, while specifying that the demand to scale with the equity of agents $\forall i \in\{1, \ldots, N\}$ will ensure agent policies are homothetic in equity even with imperfect competition. Since the outside agents only participate in output

\footnotetext{
${ }^{10}$ See Rahi and Zigrand $(2009,2013)$ for settings in which cross-market arbitrageurs reduce mispricings in segmented markets.

${ }^{11}$ That $q_{i}(\omega) f(\cdot, \omega)$ is also bounded from below follows since the collective resources of the $N$ agents, when scaled down by their collective equity, is finite.

${ }^{12}$ Appendix B provides several examples of demand functions satisfying these properties derived from standard utility functions.
} 
claims markets, their collective final consumption is given by:

$$
c_{02}(z)=\sum_{i=1}^{N} D(i, z) .
$$

Where needed, we include outside agent $N+1$ as the first entry in consumption vector $\vec{c}_{1}$.

At $t=1$, agents fully internalize their price impact on the prices of their state-continent claims and those issued by other agents. The equilibrium concept is sequential equilibrium in demand functions competition among the large agents, in which they take the beliefs and investment, as well as the security demand, and supply schedules of other agents as given. This can be viewed as a generalization of the Nash-equilibrium-in-demand-schedules concept of Kyle (1989). Since there are strategic complementarities in investment by agents, there can potentially be multiple equilibria in this setting.

\subsection{Equilibrium Definition}

A Nash Sequential Equilibrium in Demand Schedules in this economy is a list of strategy profiles $\left\{\sigma_{i}\right\}_{i=\{1, \ldots N\}} \in \Sigma_{i}$, with policy tuples $\left(c_{i 1}, k_{i}, s_{i}, \vec{a}_{i}(z)\right) \in \sigma_{i}$, and state-contingent claims prices $\{q(i, z)\}_{i \in\{1, . ., N\}}$ such that:

- Repayment Optimality: Taking strategy profiles $\sigma_{-i}$ as given, for each realization $z$ of productivity shocks, agent $i$ finds it optimal to repay its debt

- Trading Optimality: Taking strategy profiles $\sigma_{-i}$ as given, agent $i$ finds it optimal to pursue strategy $\sigma_{i}$ at $t=1$, where $\sigma_{-i}$ are the strategy profiles of the other $N-1$ agents

- Sequential Rationality: Given its trading policies encoded in $\sigma_{i}$ at $t=1$, agent $i$ finds it optimal to repay its debt at $t=2$

- Market-clearing: At date 1, the markets for all agents' state contingent claims clears, such that there is no excess demand:

$$
D(i, z)+\sum_{j=1} a_{j}(i, z) \leq 0
$$

- Consistency: All agents form rational expectations of their own strategy, and the strategies of the other agents, at all dates 
Where necessary, we refine our equilibrium concept to include Bayesian correlated equilibria, in which strategy profiles $\sigma$ can condition on an additional nonfundamental state $s$ that acts as a coordination device.

\section{Equilibrium}

We begin our analysis at $t=2$ after productivity shocks have been realized in the ex post financial network when agents repay their obligations given the state $z \in \Omega$. We call the interconnections of $t=2$ the "ex post" network since these links arise endogenously from actions taken at earlier dates. Our analysis of the repayment stage is similar, in spirit, for instance, to Eisenberg and Noe (2001), except that there is no default along the path of play. All proofs are relegated to the Appendix.

\subsection{Ex Post Network at $t=2$}

Our aim in this section is to explore how the ex post distribution of debt across agents informs us of which are central in two contexts. First, an agent can be central if its investment and savings decisions have a profound impact on the ex ante incentive compatibility constraints of other agents. Second, an agent can be central if its lending and borrowing behavior has the largest multiplier in generating trade within the network. We explore both concepts below, respectively.

To examine the first concept, we recognize that, although agents do not default along the equilibrium path, we can construct a repayment vector from their I.C. constraints in the spirit of Eisenberg and Noe (2001). Despite that our repayment vector is digital, since there is no recovery from default, we can also approach our fixed-point problem by applying the Knaster-Tarksi Fixed Point Theorem, suitably modifying the relevant metric, to conclude that such equilibrium repayment vectors for an arbitrary distribution of debt holdings can be ranked. Rather than ask how properties of the network lead to default and contagion, as in Eisenberg and Noe (2001), Acemoglu, Ozdaglar, and Tahbaz-Salehi (2015), and Roukny, Battiston, and Stiglitz (2018), we can now treat the repayment vector as informing us of how properties of the network tighten or slacken the incentive compability constraints of agents when they trade with each other at $t=1$. Tighter I.C. constraints hamper risk-sharing, and inhibit the efficient allocation of resources for production. Several comparative statistics 
illustrate how these constraints are affected by the investment outcomes from the trading game, and are summarized in Proposition 1.

Proposition 1 The incentive compatibility constraints of all agents are (weakly) relaxed by an increase in $Y(z) \vec{k}+R_{f} \vec{s}$ (element-by-element).

Proposition 1 illustrates how the investment in production and storage of each agent transmit to the incentive compatibility constraints, and consequently borrowing constraints, of all other agents through their network linkages. An increase in output production (stateby-state) and private storage by agents (weakly) relaxes the I.C. constraints of all agents in each state by increasing the pledgeability of their state-contingent claims. Each agent's balance sheet acts as a commitment device to repay its debts, since the potential loss of resources represents a salient cost of default.

Since the risky investment technology of an agent produces different levels of output in different states of the world, while savings, although less efficient, produces the same output in all states, it follows that these two forms of investment have very different impacts on agent I.C. constraints. As I.C. constraints are analogous to borrowing constraints at $t=1$, agents have potential incentives to distort their investment to expand their balance sheets. Based on Proposition 1, we can summarize this observation in the following corollary.

Corollary 1: At the margin, agent $i^{\prime}$ s borrowing constraint in state $z$ relaxes by $\frac{y_{i}(z)}{R_{f}}$ if agent $i$ substitutes savings for its risky production technology.

As the desire to borrow and lend depends on with whom that agent can trade, which influences each agent's (joint) production possibilities frontier, different network topologies can result in very different distortions to each agent's investment decisions. We illustrate this observation in the example in the next section.

Having identified how investment in production and storage impact agent I.C. constraints, we now focus on what can be gleaned from their positions in state-contingent claims to explore our second concept of centrality. This will help us understand what makes an agent more "central" or "systemic" in the financial network. The impact of an agent's holdings on network I.C. constraints depends on how those holdings impact the incentives for other agents to repay. For agent $i$ to be central, it must sell a lot of claims to agents who themselves also sell a lot of claims to the network. 
To help confirm this logic, we consider a measure of network "centrality", the KatzBonacich Centrality measure, for claim issuance. The Bonacich measures scales the adjacency matrix and examines its Leontief Inverse, and is meant to capture a weighted sum of the paths walked from each node with additional iterations or propagations of the network. Intuitively, if the adjacency matrix is aperiodic and irreducible, the centrality measure captures how, if a dollar is invested across agents arbitrarily at some date and reinvested, after a long period of time how much of that dollar, on average, would be effectively borrowed by each agent.

We consider the adjacency matrix $\xi \bar{A}^{\prime}$, where $\bar{A}=\sum_{z \in \mathcal{Z}} A(z) B(z)\left[\sum_{z \in \mathcal{Z}} B(z)\right]^{-1}, A(z)$ is a nonnegative matrix that has entries $A_{i j}(z)=-a_{i}(j, z) / a_{j}(j, z)$ with $A_{i i}(z)=0$, and $B(z)=-\operatorname{diag}\left(a_{j}(j, z)\right) . \bar{A}$ sums the claims issued across states, since row $i$ of $\bar{A}^{\prime}$ records the total nominal holdings of agent $i$ of the debt of each other agent across all states, and $\xi$ acts an economically meaningful tuning parameter for the Leontief Inverse $\left(I_{N}-\xi \bar{A}^{\prime}\right)^{-1}$ in the Bonacich Centrality measure. The total net debt of agents is also, in principle, observable.

To develop intuition, we can solve for the Katz-Bonacich Centrality $\vec{v}=\left(I_{N}-\xi \bar{A}^{\prime}\right)^{-1} \iota$ explicitly for the special case of three agents:

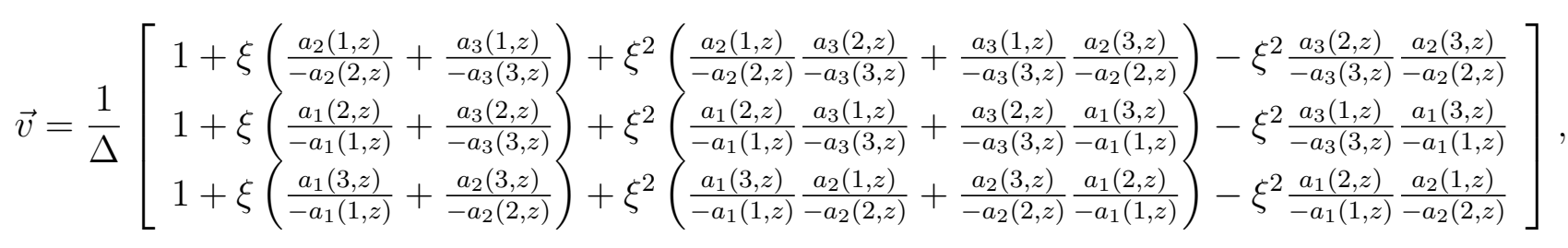

where $\Delta$ is the determinant of $I_{N}-\xi \bar{A}^{\prime}$.

The centrality measure $v_{i}$ for agent $i$ is increasing in not only the claims that $i$ issues, but also the extent to which its creditors issue claims, captured through the interaction terms $a_{j}(i, z) a_{k}(j, z)$. For instance, $a_{2}(1, z) a_{3}(2, z)$ captures the claims agent 1 issues to 2 scaled by the claims that 2 issues to 3 , which can be thought of as the indirect portion of agent $1^{\prime} s$ claims purchased by agent 3. This supports our intuition for what makes an agent a central borrower in the network. Furthermore, buying claims decreases agent $i^{\prime} s$ centrality, since it contributes to the centrality or influence of the other agents. This can be seen since agent $i^{\prime} s$ purchases $a_{i}(j, z)$ contribute to the centrality measures for agent $j$.

Which agents are effectively the largest borrowers in the network is important because such borrowers have the largest impact on the I.C. constraints of other agents through their issuance of state-contingent claims. Their claims serve as effective pledgeable capital that 
their creditors can pledge to relax their own borrowing constraints. Consequently, tightening their I.C. constraints, which reduces their state-by-state leverage, has the largest impact on tightening the constraints of their creditors, who now have less assets to lose in liquidation. This tightens the I.C. constraints of creditors, and consequently the I.C. constraints of their creditors. Through such a channel, these central agents have the largest impact on the scale of lending and intermediation in the network.

\subsection{Trading Game at $t=1$}

We now turn to the trading game at $t=1$. Consider the optimization problem for agent $i$ :

$$
\begin{aligned}
U_{1}^{i}\left(e_{i}, \vec{e}_{-i}\right) & =\sup _{\left\{c_{i 1}, k_{i}, s_{i}, d_{i}, \vec{a}_{i}(z)\right\}} u_{1}\left(c_{i 1}\right)+\sum_{z \in \mathcal{Z}} \pi(z) u_{2}\left(c_{i 2}(z)\right) \\
\text { s.t. } & : \quad c_{i 1}+k_{i}+\sum_{z \in \mathcal{Z}} \vec{a}_{-i}(z)(\vec{q}(z))+s_{i} \leq e_{i}-\sum_{z \in \mathcal{Z}} q_{i}(z) a_{i}(i, z), \\
& : \quad c_{i 2}(z)=y_{i}(z) k_{i}+\vec{a}_{-i}(z) \iota+R_{f} s_{i}+a_{i}(i, z) \\
: & \left.-a_{i}(i, z) \leq \xi\left(y_{i}(z) k_{i}+\vec{a}_{-i}(z) \iota+R_{f} s_{i}\right) \quad \text { (I.C. }\right) .
\end{aligned}
$$

Similar to Alvarez and Jermann (2000) and Hellwig and Lorenzoni (2009), the I.C. constraints of agents enter as solvency constraints in their trading game program. As agent utility is homogeneous of degree $\gamma$, and the outside trader demand scales with total agent equity, it follows that these solvency constraints can be be interpreted as state-by-state leverage constraints, in equilibrium, where $d_{i}(z) / e_{i}$ is bounded from above ${ }^{13}$

Let the Lagrange multiplier on the leverage constraint of agent $i$ in state $z$ be $\pi(z) \psi_{i}(z) e_{i}^{\gamma}$. Our analysis of the trading equilibrium gives rise to the following proposition.

Proposition 2 There exists an equilibrium in which: 1) the optimal policies of agent $i$ satisfy $c_{i 1}=\hat{c}_{i 1} e_{i}, k_{i}=\hat{k}_{i} e_{i}, a_{i}(i, z)=\hat{a}_{i}(i, z) e_{i}, a_{i}(j, z)=\hat{a}_{i}(j, z) e_{i}$, and $s_{i}=\hat{s}_{i} e_{i}$, where $\hat{c}_{i 1}, \hat{k}_{i}, \hat{s}_{i}, \hat{a}_{i}(i, z)$, and $\hat{a}_{i}(j, z)$ satisfy the equilibrium conditions ${ }^{14}$

\footnotetext{
${ }^{13}$ The homotheticity of the utility functions ensures that the policies of all agents scale linearly with their equity, which allows us to transform the state space into equity shares, which lies along the $N$ dimensional simplex, and the space of controls into fractions of equity.

${ }^{14}$ The derivatives $\frac{\partial q_{i}(z)}{\partial \hat{d}_{i}(z)}$ and $\frac{\partial q_{j}(z)}{\partial \hat{b}_{i j}(z)}$ are often referred to as the "perceived" price impact of agent $i$ in the literature on imperfect competition in financial markets. This can lead to multiple equilibria, since rational expectations requires only that the perceived be the actual impact (at least locally). Since our pricing functionals makes explicit how the demand (supply) of agent $i$ affect prices, we avoid these coordination issues arising from beliefs.
} 


$$
\begin{aligned}
\hat{k}_{i} & : \sum_{z \in \mathcal{Z}} \Lambda_{i}(z) y_{i}(z) \leq 1\left(=\text { if } \hat{k}_{i}>0\right), \\
\hat{s}_{i} & : \sum_{z \in \mathcal{Z}} \Lambda_{i}(z) \leq \frac{1}{R_{f}}\left(=\text { if } \hat{s}_{i}>0\right), \\
\hat{a}_{i}(i, z): & q_{i}(z) \leq \Lambda_{i}(z)-h_{i} \frac{\partial q_{i}(z)}{\partial \hat{a}_{i}(i, z)} \hat{a}_{i}(i, z) \quad\left(=\text { if } \hat{a}_{i}(i, z) \in\left(\hat{\bar{a}}_{i}(i, z), 0\right)\right), \\
\hat{a}_{i}(j, z): & q_{j}(z) \geq \Lambda_{i}(z)-h_{i} \frac{\partial q_{j}(z)}{\partial \hat{a}_{i}(j, z)} \hat{a}_{i}(j, z) \quad\left(=i f \hat{a}_{i}(j, z)>0\right),
\end{aligned}
$$

where $\Lambda_{i}(z)=\pi(z) \frac{u_{2}^{\prime}\left(\hat{c}_{i 2}(z)\right)+\xi \psi_{i}(z)}{u_{1}^{\prime}\left(\hat{c}_{i 1}\right)}$ is the effective state price of agent $i$ in state $z, h_{i}=$ $e_{i} / \sum_{j=1}^{N} e_{j}$, and $\widehat{\bar{a}}_{i}(i, z)$ is given in the Appendix; 2) the equilibrium price of the claim of agent $i$ issued against state $z$ can be expressed as:

$$
q_{i}(z)=f^{-1}\left(-\sum_{j=1}^{N} h_{j} \hat{a}_{j}(i, z), z\right)
$$

where $\frac{\partial q_{j}(z)}{\partial \hat{a}_{i}(j, z)},-\frac{\partial q_{i}(z)}{\partial \hat{a}_{i}(i, z)}<0 ;$ and 3) the indirect continuation utility of agent $i$ can be expressed as:

$$
U_{1}^{i}\left(e_{i}, \vec{e}_{-i}\right)=\tilde{U}_{1}^{i}\left(h_{i}, \vec{h}_{-i}\right) e_{i}^{1-\gamma}
$$

Proposition 2 reveals that the optimal choices of capital and state contingent claims satisfy Euler Equations, with the latter being modified to reflect that the strategic agents internalize their impact on asset prices. As a result of limited commitment, the effective state price of agent $i$ reflects both its marginal utility and the shadow cost of the leverage constraint. Intuitively, when an issuer's leverage constraint binds, the issuer is constrained from selling additional claims, and this short-sale constraint inflates asset prices.

The leverage constraint raises the value of capital, storage, and purchased debt when it binds to impart an endogenous collateral premium, which is absent in Alvarez and Jermann (2000), Hellwig and Lorenzoni (2009), and Chien and Lustig (2010). It arises since there is a scarcity of pledgable assets that can be produced. For instance, buying more of an agent's claims in state $z$ relaxes the I.C. constraint for that state by a fraction $\xi$ of the purchase, inflating the price of the claim because of its dual use as insurance and a pledgeable asset when buyers are constrained. Such a channel will be relevant when we discuss indirect intermediation with incomplete networks in the sequel, in which an agent has an incentive 
to be both a buyer and a seller of a claim (to different counterparties) for a given state.

We can further establish conditions under which autarky is always an equilibrium in the trading game. Similar to Alvarez and Jermann (2000), with appropriately chosen prices, agents do not have incentive to trade away (locally) from the autarky allocation, despite the wedge in their valuations. These prices ensure that there is no excess demand for statecontingent securities. Since we have outside investors, however, we also require that the implied state price pinned down by the demand of these outside investor also coincide with the autarkic allocation. This gives rise to the following corollary.

Corollary 2: Autarky is an equilibrium of the trading game iff the price of state-contingent claims at zero issuance satisfies:

$$
q_{i}(z)=\max _{j \in\{1, \ldots, N\}} \pi(z) \frac{u_{2}^{\prime}\left(c_{j 2}^{a u t}(z)\right)}{u_{1}^{\prime}\left(c_{j 1}^{a u t}\right)} \forall(i, z) .
$$

As a consequence of market power, if agent $i$ sells claims on state $z$, it shades down its supply to set marginal cost, its state price for state $z$, equal to marginal revenue, $q_{i}(z)-$ $\frac{\partial q_{i}(z)}{\partial \hat{a}_{i}(i, z)} \hat{a}_{i}(i, z)$, instead of price $q_{i}(z)$ to garner a higher valuation on its debt. Similarly, when $j$ buys agent $i^{\prime} s$ debt, it shades down its demand as a monopsonist to lower the price at which it buys $i^{\prime} s$ debt. Both forces result in less state contingent claims being traded compared to a competitive environment with the same level of capital and storage. Similar to limited commitment, market power hampers risk-sharing, but distinct from limited commitment, it also introduces cross-sectional dispersion in state prices $\left\{\Lambda_{i}(z)\right\}_{i=1}^{N}$.

Despite that financial markets are complete, agents price assets with different stochastic discount factors, a feature endemic to incomplete markets models with heterogeneous agents, such as Constantinides and Duffie (1996). Reminiscent of Constantinides and Duffie (1996), financial markets appear extremely incomplete, as dispersion in each state price across agents implies that they do not have access to any Arrow-Debreu security in a competitive setting. This wedge reflects that the limited risk-sharing stemming from market power arises from a voluntary shading of asset demand, rather than a restriction imposed by a binding leverage constraint. While limited commitment leads to imperfect risk-sharing, in which state prices but not MRSs are aligned across agents state-by-state, market power, by introducing rents, leads to incomplete risk-sharing, in which state prices are also dispersed.

As a result of the ability to exert market power in thin markets, agents eschew potential 
risk-sharing opportunities and voluntarily misalign their state prices to exploit zero-sum profit opportunities with each other. From the perspective of an outside who does not take this into account, financial markets would appear extremely incomplete, and a Social Planner with limited information would incorrectly assume that opening new markets would be a welfare-improving policy.

The next proposition introduces two notions of equivalence of our trading game equilibrium with that of incomplete markets with competitive agents. The first takes as given the equilibrium distribution of investment across agents, and characterizes the maximal asset span that would not generate trade. The second imposes the additional constraint that the incomplete market structure must also replicate the trades in the Nash Equilibrium.

Proposition 3 Let the implied state price deflator of agent $i$ in state $z$ be

$$
\Lambda_{i}(z)=q(j, z)+\frac{\partial q(j, z)}{\partial a_{i}(j, z)} a_{i}(i, z) \forall(i, j, z) .
$$

Define $\mathcal{M}$ to be the $N \times K$ matrix with elements $\mathcal{M}_{i z}=\Lambda_{i}(z)$ for agent $i$ and state $z, I_{M}$ be the $M \times M$ identity matrix, and $\iota_{N}$ to be the $N \times 1$ vector of ones. Then, there exists a market structure with $M<|\mathcal{Z}|$ assets, indexed by the $M \times K$ dividend yield matrix of the traded assets $\frac{D}{p}$, where

$$
\left(I_{M} \otimes \mathcal{M}\right) \operatorname{vec}\left(\frac{D}{p}\right)=\operatorname{vec}\left(\iota_{N} \iota_{M}^{\prime}\right)
$$

that replicates the asset span in Proposition 2. If, in addition

$$
\vec{q}=\frac{D}{p}\left(\frac{D^{\prime}}{p} \frac{D}{p}\right)^{-1} \iota
$$

then the incomplete market structure replicates the Nash Equilibrium.

The first part of Proposition 3 reveals that the intentional mispricings arising from imperfect competition are isomorphic to forced mispricings that arise from missing markets with perfect competition. As this is reflected in prices, it is possible to infer the role of market power from the extent of the implied market incompleteness, and such effects will be more pronounced when trading with larger agents that internalize their role in pricing. The wedges in state prices also suggest, in contrast to Alvarez and Jermann (2000), that the recovered state contingent claims prices cannot be used to recover the price of any nontraded, albeit redundant, security. Similar to incomplete markets, the dispersion of agent 
state prices implies that they can have different valuations for non-traded assets, despite markets being complete.

While the first notion provides some measure of the degree of implied market incompleteness from the reduced asset span, the second notion imposes that the fictitious market structure also replicates the allocations of the trading game equilibrium. The additional imposition of market-clearing to determine prices is a stricter requirement, and consequently no such incomplete markets economy may not exist. In addition, the implementation also assumes the outside agents, on net, absorb the same demand for securities as before. This second notion does, however, provide additional insight into the nature of the equilibrium by revealing what securities agents are effectively trading with each other.

We now elaborate on the role of imperfect competition in trading on risk-sharing and production in the economy, as well as how it interacts with our other key strategic friction, limited commitment. As a result of market power, agents intentionally under-insure to profit from their trading. This exposes them more to their idiosyncratic production outcomes than in a competitive setting and, because they are risk averse, this lowers their investment in capital for agents who would invest in a competitive setting. It also can lead to cross-sectional misallocation, as less productive agents may start to produce as they have less ability to invest in other agents.

Our model, consequently, highlights an investment externality from imperfect competition in financial markets. It further predicts that, as the finance industry becomes further concentrated, investment productivity in the real economy should fall as a consequence of increased voluntary exposure to idiosyncratic balance sheet risk. Since market power reduces the amount that agents borrow against each state, nominal borrowing constraints are less likely to bind, and consequently, while both limited commitment and market power reduce risk-sharing, these effects are more substitutes than complements. Both forces reduce the marginal utility of agents in states against which they would like to borrow, and raises them against states in which they would like to lend. These observations are summarized in the following proposition.

Proposition 4 As a result of market power: 1) if agent $j$ sells debt to i in state $z$, then:

$$
q_{i}(z)>q_{j}(z)
$$


and:

$$
\Lambda_{i}(z)>\Lambda_{j}(z)
$$

and market power hinders risk-sharing between $i$ and $j$ above and beyond the impact of limited commitment; 2) agents that (do not) invest in capital in a competitive setting choose a smaller (larger) scale of production $k_{i}$; 3) leverage constraints from limited commitment are less likely to bind; and 4) agent $i$ earns excess profit, $\Pi_{i}(z)$, per dollar of equity from trading securities in state $z$ :

$\Pi_{i}(z)=r_{i} h_{i} \sum_{j}\left(\frac{\partial q_{i}(z)}{\partial \hat{a}_{i}(i, z)} \hat{a}_{i}(i, z)^{2}+\frac{\partial q_{j}(z)}{\partial \hat{a}_{i}(j, z)} \hat{a}_{i}(j, z)^{2}\right)+\left(1-\frac{\Lambda_{i}(z)}{\sum_{z \in \mathcal{Z}} \Lambda_{i}(z)}\right) \sum_{j=1}^{N} \hat{a}_{i}(j, z)$,

where $r_{i}=\frac{1}{\sum_{z \in \mathcal{Z}} \Lambda_{i}(z)}$ is the effective interest rate for agent $i$. The first (nonnegative) term is its rent from market power, and is increasing in $\hat{a}_{i}(i, z)\left(\hat{a}_{i}(j, z)\right)$ when $q_{i}(z)\left(q_{j}(z)\right)$ is convex in net demand. The second term arises from risk-sharing, is positive if $i$ is a net lender $\left(\sum_{j=1}^{N} \hat{a}_{j}(i, z)>0\right)$, and is smaller if $i^{\prime} s$ debt constraint binds.

Proposition 4 also reveals that market power introduces rents from securities trading. As a consequence, there is potential economies of scale in lending, both across counterparties and states of nature. The requirement that the price function be convex in net demand is satisfied (globally) when the price function is linear. It is satisfied (locally) for the other examples given in Appendix B when a risk premium is embedded in the price and, consequently, indirect intermediation is likely to be most profitable when trading claims corresponding to high marginal utility states of nature. In addition, an agent may use storage to increase the pledgeability of its own claims across all states, leading to an inefficient distortion to production.

In addition, we can characterize the riskless rate and expected excess returns to agents from trading state contingent claims. Given that market power precludes the alignment of state prices across agents, we construct effective riskless rates from averaging their claims prices as the effective price, and their state prices as their effective benchmark riskfree return. We can then decompose the average expected excess return of debt contingent on state $z$ into three components: a risk premium that reflects the covariance between the Arrow-Debreu security and the average marginal utility of purchasing agents, a downward bias that reflects the average market power of the agents that sell claims, and a collateral premium for the 
pledgeability of debt referencing state $z$ when buyers are themselves borrowing constrained. This is summarized in the following proposition.

Proposition 5 Let $\frac{1}{r^{*}}=\sum_{z \in \mathcal{Z}} E^{*}\left[\Lambda_{i}(z)\right]$ be the state-price implied riskless rate, where $E^{*}[\cdot]$ is the cross-sectional average across its agents. Then, 1) the average expected excess payoff on debt referencing state $z R(z)=E\left[\delta_{j}(z)\right]-r^{*} E^{*}\left[q_{j}(z)\right]$ satisfies the decomposition:

$$
R(z)=\underbrace{-\operatorname{Cov}\left(\bar{u}_{2}^{\prime}(z), \delta(z)\right)}_{\text {Risk Premium }}+\underbrace{\overline{m k t}(z)}_{\text {Avg. Market Power }}+\underbrace{-\operatorname{Cov}(\overline{\operatorname{Lev}}(z), \delta(z))}_{\text {Avg. Collateral Premium }},
$$

where $\overline{m k t}(z)=-r^{*} E^{*}\left[h_{i} \frac{\partial q_{i}(z)}{\partial \hat{a}_{i}(i, z)} \hat{a}_{i}(i, z)\right] \leq 0$, and $\bar{u}_{2}^{\prime}(z)$ is the average marginal utility and $\overline{L e v}(z)$ the average leverage premium, both given in the Appendix; 2) it is sufficient, although not necessary, that:

$$
E\left[E^{*}\left[u_{2}^{\prime}\left(\frac{c_{i 2}(z)}{c_{i 1}}\right)\right]\right] \geq E\left[u_{2}^{\prime}\left(\frac{E^{*}\left[c_{i 2}(z)\right]}{E^{*}\left[c_{i 1}\right]}\right)\right]
$$

for $r^{*} \geq R_{f}$ to be lower than in the first-best economy; and 3) the market-implied riskless rate from averaging over all state contingent securities, $\left[\sum_{z \in \mathcal{Z}} E^{*}\left[q_{j}(z)\right]\right]^{-1}$, is biased downward from $r^{*}$ based on the average market power exerted in financial markets, $\sum_{z \in \mathcal{Z}} \overline{m k t}(z)$.

The proposition also reveals two observations: 1) strategic interaction affects the riskless rate implied by averaging the sums of the state prices of agents, $r^{*}$; and 2) the marketimplied riskless rate from buying a full set of state contingent claims, $\left[\sum_{z \in \mathcal{Z}} E^{*}\left[q_{j}(z)\right]\right]^{-1}$, is lower than $r^{*}$ because of market power. The latter arises because agents exercise monopoly power, and restrict supply of their securities to earn a profit from the trade. The former can lower the riskless rate below that in the first-best economy if the expected average growth rate of the marginal utility of consumption is (weakly) greater than that of the growth rate of average consumption ${ }^{15}$ This condition is reminiscent of the pricing kernel condition in Constantinides and Duffie (1996) and of the comparison in Brav, Constantinides, and Geczy (2002) of the incomplete markets SDF of heterogeneous households to its complete markets counterpart.

Finally, we briefly comment on normative implications. Although a comprehensive normative analysis is difficult in this setting, as we face not only an endogenous participation

\footnotetext{
${ }^{15}$ With idiosyncratic shocks to consumption growth across agents that are multiplicative and i.i.d. lognormally distributed, as in Constantinides and Duffie (1996), our sufficient condition always holds. Dispersion of marginal utility in their setting, however, results from market incompleteness.
} 
constraint, as in Hellwig and Lorenzoni (2009), but also multiple initial goods (consumption, capital, and storage), we can assert several normative implications. These are summarized in the following proposition.

Proposition 6 While the decentralized trading game cannot achieve the first-best allocation, it is constrained Pareto efficient when markets are competitive. It is, however, generically constrained Pareto inefficient with imperfect competition.

Proposition 6 reveals that some agents must be exposed to agent-specific risk in equilibrium, and therefore agent-specific risk can be priced even if it would vanish in the aggregate. Consequently, the trading equilibrium cannot replicate a complete markets equilibrium with perfect risk-sharing. In addition, the proposition reveals that the economy is also not constrained efficient, specifically as a result of market power. While the distortion of market power to prices does not violate constrained Pareto efficiency, its impact on agent state prices does. Since state prices are now dispersed among agents, there can be Pareto improving policies a Planner can implement to improve upon the trading game outcome. This suggests a potential role for government intervention as the market among agents becomes further concentrated.

An immediate consequence of Proposition 6 is that the distribution of productivity across agents matters for capital allocation and risk-sharing. Since agents face endogenous frictions in reallocating resources to the most efficient technologies, this misallocation is reflected in investment, asset prices, and effective aggregate productivity. In contrast, when agents are perfectly competitive and have commitment, then the competitive equilibrium achieves the first-best allocation, and only the most efficient productivity technologies are relevant for equilibrium outcomes. We summarize these observations in Corollary 3.

Corollary 3: With limited commitment and/or market power, the distribution of productivity across agents matters for the level of investment and asset prices in the economy. With commitment and perfectly competitive agents, in contrast, equilibrium outcomes depend on only the most efficient production technologies.

Corollary 3 highlights the rich interaction between market power, borrowing constraints, and investment that is absent from endowment economies with limited commitment. Alvarez and Jermann (2000), for instance, highlight an irrelevance result in which risk premia are identical with and without borrowing constraints when agent-specific and aggregate shocks 
are independent. ${ }^{16}$ In our setting, cross-sectional capital misallocation arising from limited commitment and market power distorts the production possibilities frontier. As a consequence, agent heterogeneity has a first-order impact on investment and asset prices.

\subsection{Example Economy with Three Agents}

We now study a simple example economy with linear price impact to showcase the model's fundamental mechanisms. Let there be three symmetric types of agents indexed by $i=1,2,3$ with endowments $e_{i}=e$ for all $i$ and $u_{i}\left(c_{i}\right)=\log (c)$. There are three aggregate states, $\mathcal{Z}=\{1,2,3\}$, with $y_{i}(z)=y \cdot \mathbb{1}(z=i)$ and $\pi(z)=\pi=\frac{1}{3}$, so that there exists a single agent type whose risky investment pays off in any given state. The risky technology's expected return exceeds the return on storage, $\pi y>R_{f}$.

The total mass of each agent type is equal to one, and is evenly distributed over $\frac{1}{\mu}$ symmetric members. Hence each agent of a given type has mass $\mu \in[0,1]$. (If $\mu=0$, there is a continuum of each type). We let $a_{j, n}(i, z)$ denote the asset holdings of individual agent $n$ of type $j$ for security $(i, z$,$) . Note that, given symmetry in production technologies, it is$ without loss to assume that all agents of a given type issue the same securities. The total asset holdings of $(i, z)$ by agents of type $j$ are $a_{j}(i, z)=\sum_{n} \mu \cdot a_{j, n}(i, z)$. By symmetry, take as given that $a_{j, n}(i, z)=a_{j, n^{\prime}}(i, z)=a_{j}(i, z)$ for all $n, n^{\prime}$. The outside agent's demand for security $(i, z)$ is $D(i, z)=\frac{1}{\lambda}\left(\frac{1}{y}-q(i, z)\right)$, where $\lambda$ is the inverse demand elasticity. Market clearing implies that the pricing equation and an individual agent's price impact for security $(i, z)$ is

$$
q(i, z)=\frac{1}{y}+\lambda\left(\sum_{j} a_{j}(i, z)\right) \quad \text { and } \quad \frac{\partial q(i, z)}{\partial a_{j, n}(i, z)}=\lambda \mu
$$

Going forward, it is without loss to refer to a representative agent of type $i$ with market power $\mu$. The intercept $\frac{1}{y}$ ensures that there are no arbitrage opportunities when trading with the outside agent.

To clarify the role of market power, we consider two market structures: a complete network in all representative agents form links with all other representative agents, and a core-periphery network in which a unique type is a core trader that intermediates between the other types.

\footnotetext{
${ }^{16}$ Intuitively, the unconstrained agents that can perfectly share risk are marginal in determining asset prices state-by-state, while the constrained agents that imperfectly share risk are limited in short-selling the assets that they perceive to be overvalued.
} 
We begin with the complete network (all agents can trade assets with all other agent types). Given that $i$ 's investments in the risky asset pay off if and only if $z=i$, we can take as given that $i$ issues claims against state $i$ only. This is because symmetry implies that it is never optimal to borrow against the risk-free asset, and because an agent is sure to default in states $z \neq i$. Then $a_{i}(j, z)=0$ for all $j \neq z$ and $a_{i}(i, i)<0$. The budget constraint is $k_{i}+s_{i}+\sum_{z^{\prime}} q\left(z^{\prime}, z^{\prime}\right) a_{i}\left(z^{\prime}, z^{\prime}\right)=e$, and consumption in state $z$ is $c_{i}(z)=$ $y_{i}(z) e+a_{i}(z, z)-y_{i}(z) \sum_{z^{\prime}} q\left(z^{\prime}, z^{\prime}\right) a_{i}\left(z^{\prime}, z^{\prime}\right)+\left(R_{f}-y_{i}(z)\right) s_{i}$. Limited commitment implies that each agent can default on his debt, but incurs a deadweight cost of a fraction $\xi$ of his remaining consumption in doing so. To prevent default by agent $i$, we must therefore satisfy the constraint $(1-\xi)\left(c_{i}(i)-a_{i}(i, i)\right) \leq c_{i}(i)$ This can be rearranged to give the borrowing constraint

$$
a_{i}(i, i) \leq\left(\frac{\xi}{1-\xi y q(i, i)}\right)\left(y e-\left(y-R_{f}\right) s_{i}-y \sum_{z^{\prime} \neq i} q\left(z^{\prime}, z^{\prime}\right) a_{i}\left(z^{\prime}, z^{\prime}\right)\right)
$$

The decision problem is $\max _{\vec{a}_{i}, s_{i}} \sum_{z} \pi(z) u\left(c_{i}(z)\right)$ subject to (3). Note that the borrowing constraint tightens in $s_{i}$ but slackens in $q(i, i)$. We look for a symmetric equilibrium in which $a_{i}(i, i)=-a_{s}$ and $a_{i}(j, j)=a_{b}$ for all $i$ and $j \neq i$. This implies a symmetric asset price $q(i, i)=\frac{1}{y}+\lambda\left(2 a_{b}-a_{s}\right)$ for all $i$. Moreover, consumption does not vary across states in which the agent's investments do not pay off, $c_{i}(z)=c_{i}\left(z^{\prime}\right)=c^{-}$for all $z, z^{\prime} \neq i$, but may differ across states with and without production, $c^{-} \leq c_{i}(i)=c^{+}$. The relevant marginal rate of subsitution is $\Lambda=\frac{u^{\prime}\left(c^{-}\right)}{u^{\prime}\left(c^{+}\right)}$. (Note that $\Lambda=1$ in the first-best allocation.) To gain intuition, assume first that borrowing constraint (3) does not bind. The first-order conditions for $a_{s}, a_{b}$ and $s$ then are, respectively,

$$
\begin{array}{r}
\pi u^{\prime}\left(c^{+}\right)=\pi u^{\prime}\left(c^{+}\right) y\left(q^{*}-\mu \lambda a_{s}\right) . \\
\pi u^{\prime}\left(c^{-}\right)=\pi u^{\prime}\left(c^{+}\right) y\left(q^{*}+\mu \lambda a_{b}\right) . \\
2 \pi u^{\prime}\left(c^{-}\right) R_{f}-\pi u^{\prime}\left(c^{+}\right)\left(y-R_{f}\right) \leq 0 .
\end{array}
$$

where the last condition holds with equality if $s>0$. This is a system of equations that can be solved in closed form, and yields the following result.

Proposition 7 If the borrowing constraint is slack, the complete network equilibrium is such that $q^{*}=\frac{1}{y}+\frac{2 \mu \lambda a_{b}}{1+\mu}$ and $\Lambda=1+\frac{\mu \lambda y(3+\mu) a_{b}}{1+\mu}$. For any $\lambda>0$, the equilibrium allocation is equal 
to the first-best allocation if and only if agents do not have any market power $(\mu=0)$.

When agents have market power $(\mu>0)$, the first-order conditions reveal that agents shade down purchases in order to lower asset prices, and shade down sales to raise asset prices.

While internalizing price impact is privately optimal, it imposes externalities on the other side of the market. Specifically, it is more costly to obtain insurance if sellers restrict supply to raise prices. Likewise, restricting demand to lower prices reduces the amount of capital that can raised through asset sales, tightening the budget constraint. Both forces combine to inefficiently distort production, and leads to lower welfare relative to first-best.

Which force dominates depends on equilibrium portfolios. Given the linearity of price impact, the inframarginal benefit of distorting quantities is largest for assets in which one holds a large market share. In equilibrium, each agent thus has stronger incentives to undersupply the own asset in order to raise its price.
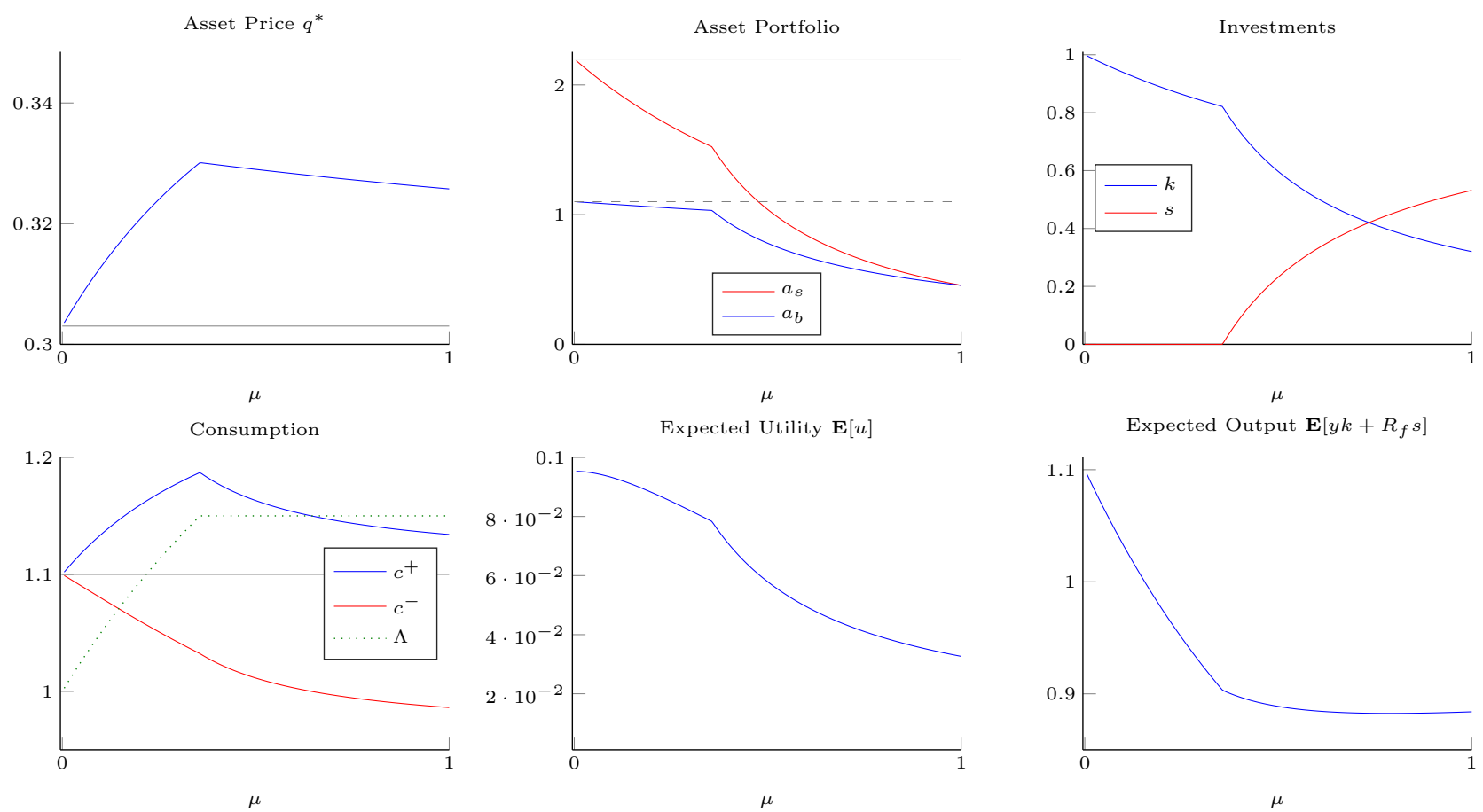

Figure 1: Equilibrium in complete network given $\mu$ assuming that the borrowing constraint does not bind. Parameters: $e=1, y=3.3, \mathbf{E}[y]=1.1, R_{f}=1, \lambda=0.05$ Gray horizontal lines show the first-best equilibrium with $q^{*}=\frac{1}{y}, a_{s}=2 \pi y e, a_{b}=\pi y e$, and $c^{+}=c^{-}=\pi y k$.

Figure 1 plots equilibrium outcomes as a function the market power parameter $\mu$. The top left panel shows the asset price, the top middle panel shows the asset portfolio and the top right panel shows investments. In the bottom row, the left panel shows the distribution 
of consumption across states, the middle panel shows expected utility, and the right panel shows expected output. Gray horizontal lines depict the first-best allocation. Internalizing price impact initially boosts the asset price because each agent sells more of his own security than he buys of the others. This hurts the buyers of the security, forcing them to cut back on risk-sharing. As a result, each agents invests less in the risky technology and begins to tolerate a consumption spread across states. The green dashed line shows this dispersion in marginal utilities across states by plotting the marginal rate of subsitution $\Lambda$. When market power is sufficiently strong (and asset prices are sufficiently high), agents begin to self-insure by investing in inefficient storage. This leads to a sharper reduction in asset purchases, lowering demand and asset prices. This reduces the returns from selling assets, leading to a sharp decline in risky investment. Coupled with an increase in storage, the resulting decline in insurance demand associated further lowers output and investment, and utility falls. In equilibrium, all agents thus suffer from the privately optimal exploitation of market power.

So far we have argued that market power alone can distort risk-sharing and investment. Next, we study the interaction of market power with binding borrowing constraints. To provide the intuition in the clearest possible manner, we begin by studying the case of a stringent borrowing constraint with $\xi=0$ that does not permit any asset issuance, $a_{s}=0$. Conditional on the (undetermined) consumption levels $c^{+}$and $c^{-}$, the first-order conditions for $a_{b}$ and $s$ are the analogues to before, i.e.

$$
\begin{gathered}
u^{\prime}\left(c^{-}\right)-u^{\prime}\left(c^{+}\right) y\left(q^{*}+\lambda \mu a_{b}\right)=0 . \\
2 \pi u^{\prime}\left(c^{-}\right) R_{f}-\pi u^{\prime}\left(c^{+}\right)\left(y-R_{f}\right) \leq 0 .
\end{gathered}
$$

where the latter condition holds with equality if $s>0$. As before, there is an incentive to shade down demand to lower asset prices. The key difference is that the binding borrowing constraint eliminates any strategic distortions when it comes to asset sales, however. As a result, the equilibrium asset price is decreasing in $\mu$.

Proposition 8 The equilibrium in complete networks with stringent borrowing constraints $(\xi=0)$ is such that $a_{s}=0, q=\frac{1}{y}+2 \lambda a_{b}$, and $\Lambda=1+(2+\mu) y \lambda a_{b}$. The first-best allocation is not attained for any $\mu$, the asset price is strictly decreasing in $\mu$, and expected utility is strictly increasing in $\mu$.

The stark difference in comparative statics stems from the fact that the borrowing constraint 
entirely reallocates de-facto market power from sellers to buyers. Hence there is no strategic undersupply of assets that might serve to boost asset prices. Figure 2 plots the associated equilibrium. All panels are plotted on the same scale as in Figure1 to facilitate comparisons. Gray horizontal lines continue to indicate the first-best allocation.
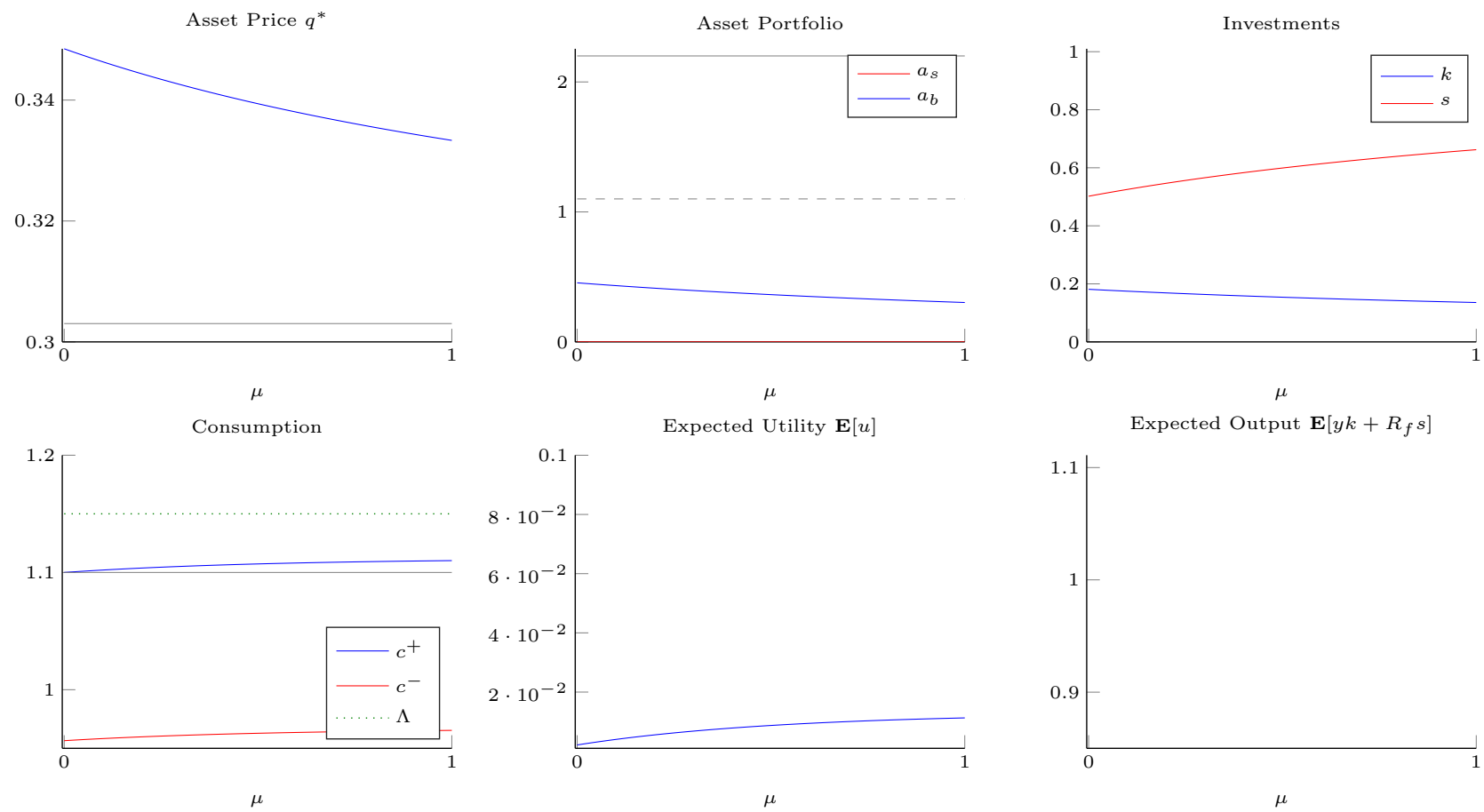

Expected Output $\mathbf{E}\left[y k+R_{f} s\right]$

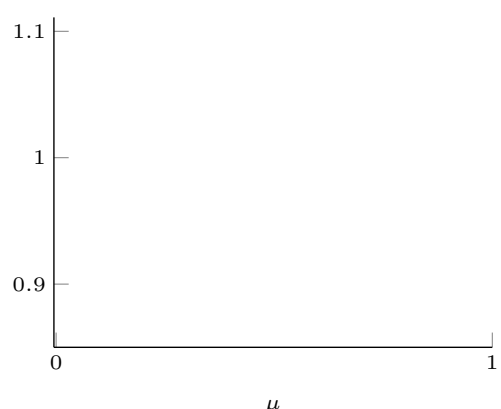

Figure 2: Equilibrium in complete network given $\mu$ with a stringent borrowing constraint $(\xi=0)$. Parameters: $e=1, y=3.3, \mathbf{E}[y]=1.1, R_{f}=1, \lambda=0.05$ Gray horizontal lines show the first-best equilibrium with $q^{*}=\frac{1}{y}, a_{s}=2 \pi y e, a_{b}=\pi y e$, and $c^{+}=c^{-}=\pi y k$.

Asset prices are higher in levels than in the absence of borrowing constraints because limited commitment lowers the supply of assets. The comparative statics differ because only buyers are strategic, hence all strategic players have an interest in lowering prices. This implies that there is no negative externality from internalizing price impact, and expected utility is increasing in market power. Utility levels are low on average because insurance is expensive, forcing agents to self-insure with inefficient storage and lowering expected output and consumption. Borrowing constraints thus substantially alter equilibrium asset prices and efficiency by redistributing strategic market power from sellers to buyers, and by constraining opportunities for risk-sharing.

Remark 9 While we have only illustrated the polar cases with a slack borrowing constraint and the stringent borrowing constraint $\xi=0$, intermediate values of $\xi$ trace out the range 
between the polar cases.

\section{Incomplete Network}

In this section, we consider an extension of our model in which agents are now part of a fixed network $\mathcal{G}$ that restricts with whom they can trade output claims. One can view this

network rigidity as reflecting the costs of opening markets and trading relationships between agents, or as the costs of doing due diligence about the creditor or borrower. This will allow us to explore how the capacity to trade across segmented markets impacts production and risk-sharing.

In this network, an undirected link $l_{i j} \in \mathcal{G}$ in this graph, with support $\{0,1\}$, implies that agent $i$ can buy $a_{i}(j, z) \geq 0$ shares of contingent from agent $j$ for state $z$ at $t=1$, and sells $a_{i}(i, z) \leq 0$ shares to other agents. Without loss for our analysis, we assume that all agents are connected to each other, either directly or indirectly through other agents. The budget constraint of agent $i$ at $t=0$ is then:

$$
c_{1 i}+k_{i}+\sum_{z \in \mathcal{Z}}\left(\vec{a}_{i}(z) \odot \vec{l}_{i}^{\prime}\right) \vec{q}(z)+s_{i} \leq e_{i}-\sum_{z \in \mathcal{Z}} q(i, z) a_{i}(i, z)
$$

where $\vec{l}_{i}$ is the digital vector that summarizes the intermediaries from whom $i$ can purchase output claims. Given its links, the optimization problem for agent $i$ is now:

$$
\begin{aligned}
U_{1}^{i}\left(e_{i}, \vec{e}_{-i}\right) & =\sup _{\left\{c_{i 1}, k_{i}, s_{i}, d_{i}, \vec{a}_{i}(z)\right\}} u_{1}\left(c_{i 1}\right)+\sum_{z \in \mathcal{Z}} \pi(z) u_{2}\left(c_{i 2}(z)\right) \\
\text { s.t. } & : \quad c_{i 1}+k_{i}+\sum_{z \in \mathcal{Z}} \vec{a}_{-i}(z) \vec{q}(z)+s_{i} \leq e_{i}-\sum_{z \in \mathcal{Z}} q_{i}(z) a_{i}(i, z), \\
& c_{i 2}(z)=y_{i}(z) k_{i}+\vec{a}_{-i}(z) \vec{l}_{i}+R_{f} s_{i}+a_{i}(i, z) \\
: & \left.-a_{i}(i, z) \leq \xi\left(y_{i}(z) k_{i}+\vec{a}_{-i}(z) \operatorname{vecl}_{i}+R_{f} s_{i}\right) \quad \text { (I.C. }\right) .
\end{aligned}
$$

The equilibrium is summarized by Proposition 2, suitably modified to reflect that agents are restricted in with whom they can trade output claims.

From Proposition 4, agents create an intermediation spread between the securities they buy and sell in each state, so that they charge a higher rate to borrowers than they pay to creditors. This can be seen from the first term in their trading profit in each state in equation (2). This provides an arbitrage opportunity in an incomplete network, as a central 
intermediary can arbitrage across segmented markets. The rent earned from intermediating funds could also potentially give rise to a core-periphery network structure when forming links is costly, as the rents from being a central agent with market power can defray the costs of paying for the additional links to indirectly intermediate funds from lenders to borrowers.

A potential tradeoff to this arbitrage, unique to our setting, however, is that it impacts the cost of acquiring insurance for the risk-averse arbitrageur. The second term in the profit expression above, while also present in a competitive setting, reflects the embedded risk premium from buying insurance, which is negative when agent $i$ is a net borrower. The need for insurance against states in which an agent itself has high marginal utility acts as an endogenous limits to arbitrage from arbitraging across segmented markets ${ }^{17}$ As a consequence, there is an interior optimum to the scale of the arbitrage for claims in states of the world in which the central agent itself needs insurance.

In addition, as a result of imperfect competition among agents in an incomplete network, gross flows are also the relevant quantity for balance sheets because of the dispersion of state prices, whereas only net flows (within a state) matter with perfect competition. Whom trades with whom is important for understanding the dispersion in asset prices on otherwise similar securities. To see this, we can express the net market value of debt of agent $i$ in state $z$ as:

$$
-\sum_{j=1}^{N} q_{j}(z) \hat{a}_{i}(j, z)=-\Lambda_{i}(z) \sum_{j=1}^{N} \hat{a}_{i}(j, z)+\sum_{j=1}^{N} \frac{\partial q_{j}(z)}{\partial \hat{a}_{i}(j, z)} \hat{a}_{i}(j, z)^{2} .
$$

While the first term, which is also present with perfect competition, depends only the the net flow, the second two terms reflect the discrepancy in the security valuations between $q_{i}(z)$ and $q_{j}(z)$ as a result of market power. These two terms depend on agent $i^{\prime} s$ specific cross-balance sheet linkages, and cannot be netted out into a net flow.

Finally, the topology of the network feeds back into the role of financial markets to efficiently share risks and allocate capital. The ability to intermediate across segmented markets is the key new force that an incomplete network introduces 18 This introduces two

\footnotetext{
${ }^{17}$ There is a vast literature on the limits to arbitrage, including, for instance, De Long et al. (1990), Xiong (2001), Gromb and Vayanos (2002), and Brunnermeier and Pedersen (2009). In contrast to these studies, it is not risk aversion, portfolio constraints, or asymmetric information that gives rise to mispricing in our setting. Instead, it is that the convergence trade for arbitraging agents worsens the price at which they acquire the security for their own consumption.

${ }^{18}$ Rahi and Zigrand $(2009,2013)$ consider endowment economies in which risk-neutral arbitrageurs strategically trade across segmented markets against competitive investors.
} 
distortions. First, by arbitraging across markets state-by-state, core agents ration credit to producers and borrower from end providers of capital. This distorts the supply of capital to productive agents. Second, since core agents face borrowing constraints, they may invest in inefficient storage to increase the pledgeability of their balance sheet in all states to be able to more efficiently arbitrage across segmented markets.

Importantly, market power is essential for network effects in pricing and risk-sharing to arise among agents. The following corollary highlights that, while the distortions from limited commitment to investment would also be present in a competitive setting, market power is essential for gross flows to matter and for double-margining.

Corollary 4: If agents behave competitively: 1) there is no indirect intermediation or network effects beyond price externalities; and 2) investment in capital is (above) below its efficient level for agents that (do not) invest in the absence of limited commitment.

We next investigate these forces by revisiting our 3 agent example, and characterizing the asymmetric equilibrium that arises with a core-periphery network structure.

\subsection{Core Periphery Example Economy with Three Agents}

To further understand the role of market power, we now study a core-periphery market structure in which a representative core agent trades with two representative peripheral agents, and the periphery trades with the core but not with each other. In contrast to the complete network, the core may now buy and issue claims against a state in which his investment does not pay off to facilitate intermediation. This opens the door to arbitrage opportunities (buying and selling claims on the same state at different prices).

We again look for an equilibrium that is symmetric conditional on the market structure. Hence the core treats the periphery symmetrically, and the periphery holds a symmetric portfolio. There are now three types of asset: the core asset issued by the core against the state in which his risky investment pays off, the periphery asset issued by each periphery agent against the state in which his asset pays off, and the arbitrage asset issued by the core against a state in which a periphery's investment pays off, and sold to the periphery agent whose investment does not pay off in that state. We index these assets and the associated states by subscripts $c, p$, and $a$, and use $\hat{a}_{z}$ and $a_{z}$ to denote core and periphery holdings of the asset associated with state $z\{c, p, a\}$. The three types of asset trade at prices $q_{c}, q_{p}$, and 
$q_{a}$.

Each periphery agent decides on its issuance of its periphery asset $a_{p}$, its purchases of the core asset $a_{c}$ and of the arbitrage asset $a_{a}$, and its saving $s$. Because there are no direct links between the periphery, it does not buy the other periphery agent's asset. Consumption may differ across all three states. Hence we will denote periphery consumption levels by $c_{c}$ (consumption when the core's investment pays off), $c_{p}$ (consumption when the own investments pay off), and $c_{a}$ (consumption when the other peripheral agent's asset pays off), respectively. The borrowing constraint is the analogue to before. If it does not bind, the first-order conditions with respect to $a_{p}, a_{a}, a_{c}$ and $s$ are, respectively,

$$
\begin{gathered}
u^{\prime}\left(c_{p}\right)=u^{\prime}\left(c_{p}\right) y\left(q_{p}-\mu \lambda a_{p}\right) \\
u^{\prime}\left(c_{a}\right)=u^{\prime}\left(c_{p}\right) y\left(q_{a}+\mu \lambda a_{a}\right) \\
u^{\prime}\left(c_{c}\right)=u^{\prime}\left(c_{p}\right) y\left(q_{c}+\mu \lambda a_{c}\right) \\
u^{\prime}\left(c_{a}\right) R_{f}+u^{\prime}\left(c_{c}\right) R_{f}-u^{\prime}\left(c_{p}\right)\left(y-R_{f}\right) \leq 0
\end{gathered}
$$

Much as before, there is an incentive to shade down asset supply and asset demand to boost $q_{p}$, the price of the issued asset and to lower $q_{c}$ and $q_{a}$, the price of the core and arbitrage asset. The difference is that there is no strategic impact on the price of the purchases peripheral asset. The first-order condition on savings binds only if insurance via asset purchases is too expensive.

The core decides on its own asset issuance $\hat{a}_{c}$, its (symmetric) holdings of each of the periphery assets $\hat{a}_{p}$, its issuance of each of the arbitrage assets $\hat{a}_{a}$, and its savings $\hat{s}$. In addition to the borrowing constraint on the own asset, the core faces a borrowing constraint on its arbitrage asset given by

$$
\hat{a}_{a} \leq \xi\left[\hat{a}_{p}+R_{f} \hat{s}\right]
$$

In a symmetric equilibrium, consumption is $c^{+}$when the core's risky investment pays off, and $c^{-}$when it does not. Asset prices satisfy

$$
q_{c}=\frac{1}{y}+\lambda\left(2 a_{c}-\hat{a}_{c}\right) \quad q_{p}=\frac{1}{y}+\lambda\left(\hat{a}_{p}-a_{p}\right) \quad q_{a}=\frac{1}{y}+\lambda\left(a_{a}-\hat{a}_{a}\right)
$$

Observe that the market structure gives rise to market size effect: because the core has two buyers for its asset, and is the unique buyer or seller of every other asset, it benefits from 
higher sale prices and can exercise more de-facto market power than periphery agents.

We develop intuition by first focusing states of the world in which the core's investment does not pay off, and he instead engages in intermediation. In any such state, the core receives assets from periphery agent whose investment pays off, and pays out claims to the other periphery. Hence $c^{-}=\hat{a}_{p}-\hat{a}_{a}+R_{f} \hat{s}$, and there is an arbitrage opportunity if $q_{p}<q_{a}$. The portfolio simultaneously determines core consumption. We therefore define the core's arbitrage problem as the decision problem that determines how to deliver a given target level of consumption $\bar{c}$ minimal cost. (The consumption target $\bar{c}$ can be solved for separately.) Given $\bar{c}$, the arbitrage problem can thus be simply states as

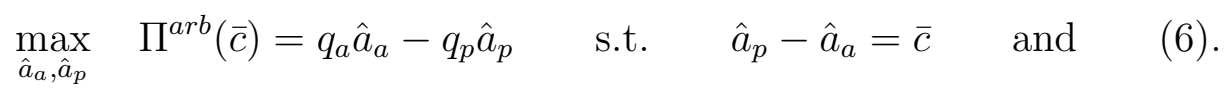

If the borrowing constraint does not bind on the arbitrage, first-order conditions reveal that the optimal arbitrage portfolio for a core trader who internalizes his price impact is

$$
\hat{a}_{a}^{*}(\bar{c})=\frac{q_{a}-q_{p}}{2 \mu \lambda}-\frac{\bar{c}}{2} \quad \text { and } \quad \hat{a}_{p}^{*}(\bar{c})=\frac{q_{a}-q_{p}}{2 \mu \lambda}+\frac{\bar{c}}{2}
$$

That is, gross positions are increasing in the arbitrage spread $q_{a}-q_{p}$, and are decreasing in market power $\mu$. The latter effect arises because the spread moves against the arbitrage whenever a trader with market power increases his position. The core's strategic restraint harms the periphery, who must sell low at $q_{p}$ and buy high at $q_{a}$. There is no arbitrage spread if and only if the core has no market power.

Proposition 10 If the borrowing constraint is slack, the core-periphery equilibrium features a strictly positive arbitrage spread $q_{a}^{*}-q_{p}^{*}$ for all $\mu>0$. The spread is zero $\left(q_{a}^{*}=q_{p}^{*}\right)$ and the equilibrium delivers the first-best allocation if and only if $\mu=0$ and $\lim \lambda \rightarrow 0$. In this limit, moreover, the core acts as a pure pass through and sets $\hat{a}_{p}=2 \hat{a}_{a}=\pi y e$.

Given the optimal arbitrage porftolio, the core's consumption in the state in which its own investment pays off can be written as

$$
\hat{c}^{+}=y e-\hat{a}\left(1-q_{c} y\right)+2 y \Pi^{*}(\bar{c})+\left(R_{f}-y\right) \hat{s} .
$$

Since arbitrage profits are decreasing in $\bar{c}$, consumption in state $c$ is strictly decreasing in $\bar{c}$, while consumption in states $z \neq c$ is strictly increasing. The first-order conditions for $\hat{a}_{c}, \bar{c}$, 
and $\hat{s}$ are

$$
\begin{aligned}
\pi u^{\prime}\left(\hat{c}^{+}\right) & =\pi u^{\prime}\left(\hat{c}^{+}\right) y\left(q_{c}-\lambda \hat{a}_{c}\right) \\
2 \pi u^{\prime}\left(\hat{c}^{-}\right) & =\pi u^{\prime}\left(\hat{c}^{+}\right) 2 y\left(-\frac{\partial \Pi^{a r b}(\bar{c})}{\partial \bar{c}}\right) .
\end{aligned}
$$

The first condition shows the standard decision problem with strategic undersupply of the own asset. The optimal choice of $\bar{c}$ resolves a trade-off between arbitrage profitability and risk-sharing.
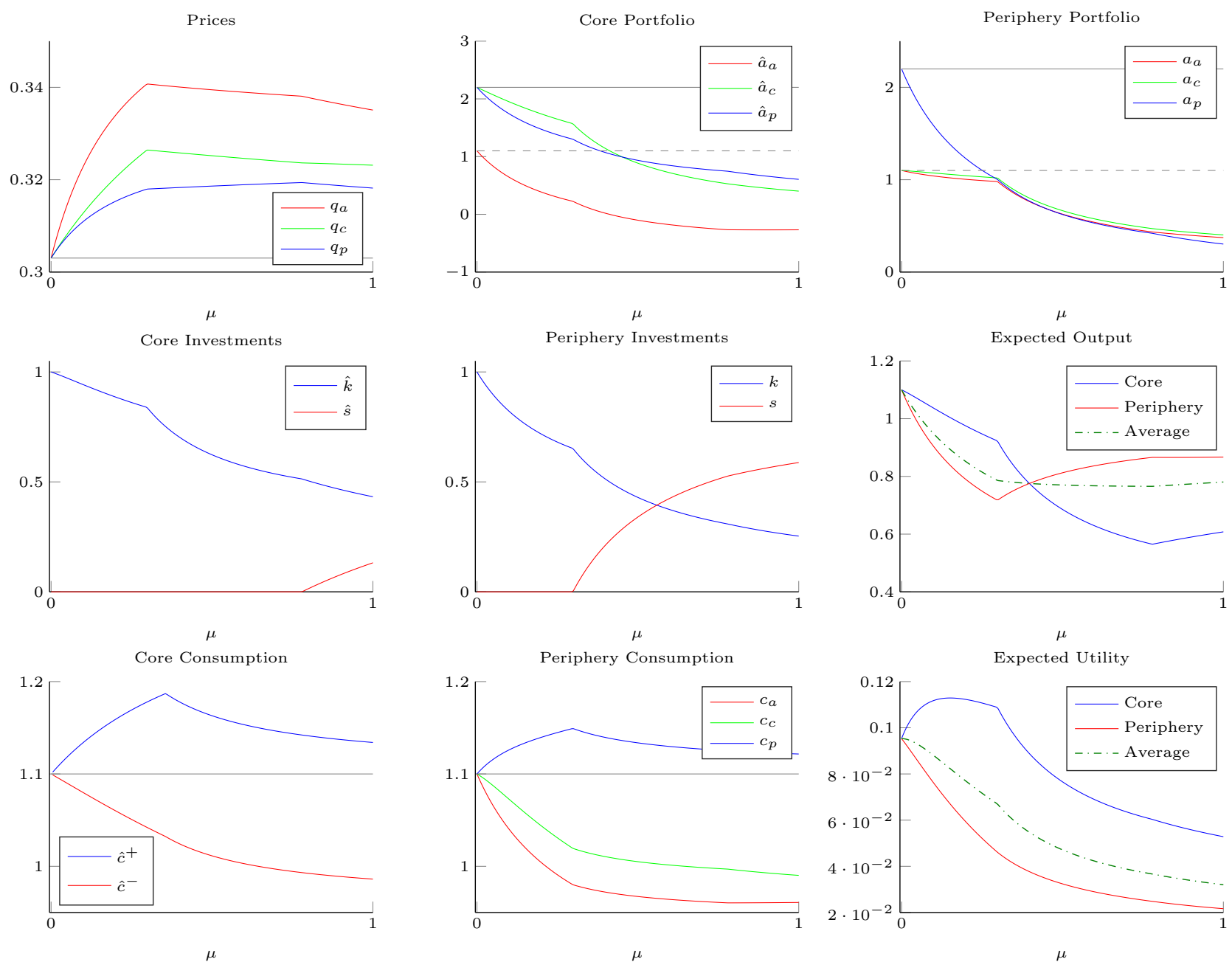

Figure 3: Equilibrium in core periphery network given $\mu$ assuming the borrowing constraint is slack. Parameters: $e=1, y=3.3, \mathbf{E}[y]=1.1, R_{f}=1, \lambda=0.05$ Gray horizontal lines show the first-best equilibrium with $q^{*}=\frac{1}{y}, a_{s}=2 \pi y e, a_{b}=\pi y e$, and $c^{+}=c^{-}=\pi y k$.

Figure 3 illustrates the resulting equilibrium. Asset prices are in the top left corner. For all $\mu>0$, there is a strictly positive arbitrage spread $q_{a}-q_{p}$. Market power initially serves 
to strengthen this arbitrage, boosting core utility at the expense of the periphery. Since insurance is expensive for the periphery, it responds by investing in storage. Because the core purposefully underprovides insurance, investment efficiency declines for all agents. This leads to a decline in average expected utility.
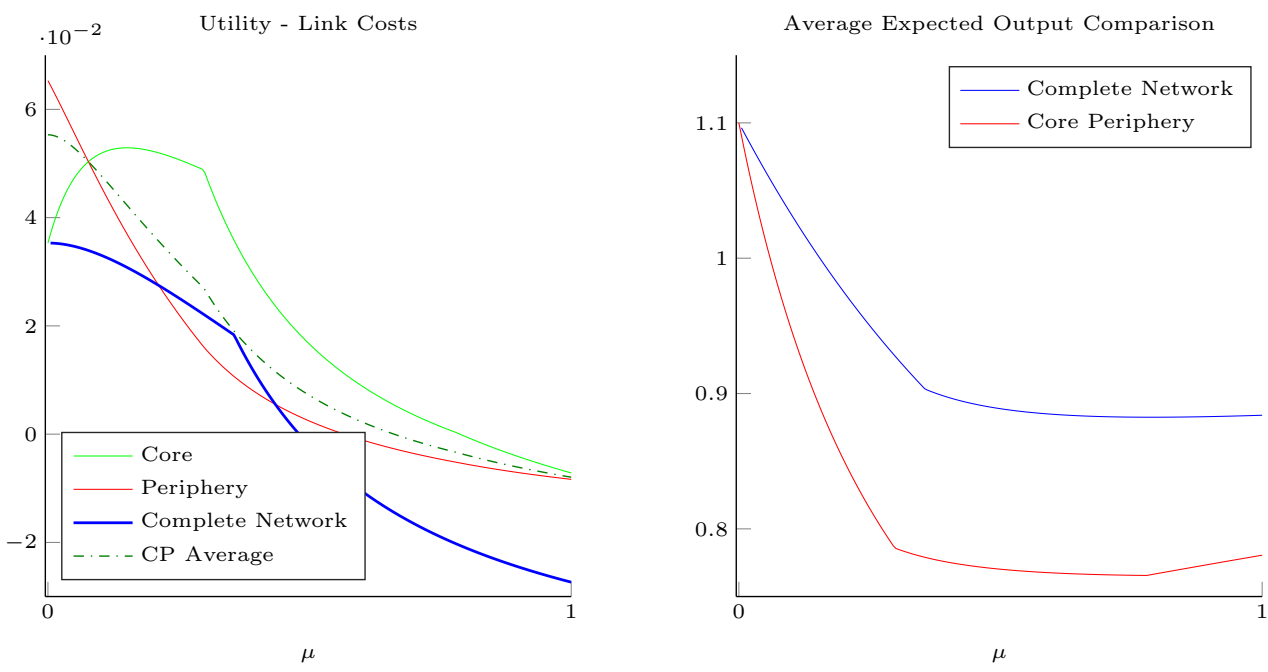

Figure 4: Utility and output comparison across market structures. Parameters: $e=1, y=3.3$, $\mathbf{E}[y]=1.1, R_{f}=1, \lambda=0.05$ Gray horizontal lines show the first-best equilibrium with $q^{*}=\frac{1}{y}$, $a_{s}=2 \pi y e, a_{b}=\pi y e$, and $c^{+}=c^{-}=\pi y k$. Link cost $\chi=0.015$.

Figure 4 shows that the presence of arbitrage rents may sustain a core-periphery network as an equilibrium outcome, despite that it lowers productive efficiency relative to the complete network. To illustrate this, we plot equilibrium utility minus a fixed cost of forming each link required to create the network structure in the left panel, and expected output in the right panel. When linking costs are relatively high, all agents may prefer a core-periphery structure to a complete network, although it generates lower output. The core is willing to pay for additional links because doing so provides arbitrage opportunities. The periphery accepts lower production efficiency in exchange for lower trading costs. The network structure thus endogenously strengthens the harmful effects of market power.

The ability to double margin lenders and borrowers can, consequently, help explain the core-periphery structure often observed in lending in financial markets outside of exchanges. This is in contrast to Farboodi (2016), in which a core-periphery structure arises because intermediation spreads decline with distance along the intermediation chain, and Craig and Ma (2018), which rationalizes it with heterogeneity across banks in project quality and link costs. 
Lastly, we discuss the effects of borrowing constraints in a core periphery. The borrowing constraint on issuances of the own asset are well understood already. Hence we focus now on a binding borrowing constraint when the core arbitrages across the periphery. If constraint (6) binds, arbitrage profits in a given state can be written as

$$
\Pi=q_{a} \xi\left[\hat{a}_{p}+R_{f} s\right]-q_{p} \hat{a}_{p}=\left(q_{a} \xi-q_{p}\right) \hat{a}_{p}+q_{a} \xi R_{f} \hat{s}
$$

Hence savings now raise arbitrage profits by relaxing the borrowing constraint, and the defacto return to savings is now $R_{f}\left(1+p a_{\xi}\right)>R_{f}$. This means that core agents are more likely to distort their investment towards inefficient savings in order to relax the borrowing constraint that limits arbitrage. Market power thus necessitates the creation of borrowing capacity. More generally, this implies that agents with low risk production capacity or commitment power are more likely to be central in a core periphery network.

\section{$5 \quad$ Empirical Implications}

Our theoretical investigation gives rise to several empirical predictions as financial markets become more concentrated. First, more downstream agents in intermediation chains are more central, in that changes in their balance sheets have the largest impact on the borrowing capacities of other agents in the economy. Second, one would expect to observe systematic under-investment in risky projects, whether it be C\&I or syndicated lending by banks, risky debt purchases by bond funds, or capital investment by firms as a result of under-insurance, for instance, in wholesale lending, REPO, and OTC markets. Furthermore, the debt of large agents and the firms in which they invest should have lower expected returns as a result of imperfect competition. Third, one would also expect a secular decline in discount rates as agents become larger and internalize their market power.

In addition, leverage constraints and market power act as substitute channels for underinsurance across agents. Since sellers benefit from market power only when they are unconstrained, leverage constraints should bind less, and consequently be less relevant, for larger agents. When sellers are unconstrained, they determine the bias in asset prices, whereas buyers' asset positions should become more relevant when sellers are constrained.

Our results also have empirical implications for intermediary asset pricing. Cochrane (1991, 1996) emphasize that production outcomes of firms should reflect the marginal rates 
of transformation (MRT) of firms maximizing shareholder value, just as consumption reflects the marginal rates of substitution (MRS) of consumers. The limitation that firms can transfer production only across time with capital investment, but not across states, however, limits the ability to recover MRTs from investment returns ${ }^{19}$ Our analysis suggests, if agents in our model can be interpreted as intermediaries, that it is can be the contracts that intermediaries write with each other that reflects state contingency in (effectively joint) production.

Recall that agent $i$ produces net output $c_{i 2}(z)$ at $t=2$ in state $z \in \mathcal{Z}$. This output reflects both agent $i^{\prime} s$ own production, net of what it sends to other agents to honor its contracts from $t=1$. These contracts can be financial securities agents trade with each other, such as collateralized debt, equity, and derivatives, or as the delivery of actual goods employable in some alternative production technology. While its choice of capital $k_{i}$ at $t=1$ suffers from intratemporal rigidity, its net output does not because of its contracts with other agents that reintroduce state contingency. Our analysis suggests that examining earnings and inter-intermediary exposures may be better indicative of not only their market power, but also intermediary state prices relevant for how they would price assets.

We can, for instance, take a Taylor expansion of the implied state price of agent $i$ around initial earnings to find that

$$
\Lambda \approx \alpha_{0}-\alpha_{1} \frac{c_{i 2}(z)}{c_{i 1}}+\alpha_{2} \tilde{\psi}_{i}(z)
$$

where $\frac{c_{i 2}(z)}{c_{i 1}}$ is earnings growth and $\tilde{\psi}_{i}(z)$ reflects capacity constraints. One can then test this SDF on test portfolios following a GMM or Fama-MacBeth approach.

Furthermore, the ability for agents to profit from double-margining when trading across segmented markets has implications for the costs of capital in the economy. As lenders internalize how they price the debt of borrowers, our model predicts increasing costs of capital as one moves downstream in the intermediation chain. End lenders, such as depositors or institutional investors, should earn lower returns than the agents who lend on their behalf to other agents or firms.

Finally, we expect to see a more (less) interconnected financial system in times of low (high) aggregate uncertainty, as the benefits from risk-sharing diminish as the commonality in they payoffs to risky investment increases. In addition, our theory can also potentially ra-

\footnotetext{
${ }^{19}$ Belo (2010), for instance, posits that it is the ability of firms to vary their productivity across states, subject to a budget constraint in reassigning conditional probabilities, that makes investment returns informative about MRTs.
} 
tionalize both core-periphery network structures and segmented markets for similar products among large agents as stemming from the tradeoffs of strategic interaction.

\section{Conclusion}

We construct a model of concentrated markets in which large, risk-averse agents with limited commitment invest in risky projects and internalize their price impact when trading contingent claims with each other. Central agents in our setting are those who issue claims to agents who themselves issue claims against output, since their production and savings decisions have the greatest impact on relaxing effective capacity constraints in the economy. As a result of strategic interaction, agents voluntarily under-insure production risk with each other. Such under-insurance adversely impacts capital investment decisions and, despite market completeness, generates dispersion in state prices. This strategic market incompleteness can lead to arbitrage opportunities across segmented markets and a decline in the risk-free rate, and can introduce a role for a government to implement welfare-improving policies. These forces feed back into the benefits for agents to trade with each other, and help rationalize core-periphery networks and market segmentation when links are costly. 


\section{References}

Acemoglu, D., A. Ozdaglar, and A. Tahbaz-Salehi (2015): "Systemic Risk and Stability in Financial Networks," American Economic Review, 105, 564-608.

Allen, F., And D. Gale (2000): "Financial Contagion," Journal of Political Economy, $108,1-33$.

Alvarez, F., And U. Jermann (2000): "Efficiency, Equilibrium, and Asset Pricing with Risk of Default," Econometrica, 68(4), 775-797.

Anthropelos, M. (2017): "The effect of market power on risk-sharing," Mathematics and Financial Economics, 11(3), 323-368.

Barattieri, A., L. Moretti, and V. Quadrini (2017): "Banks' Interconnectivity and Leverage," mimeo.

BASAK, S. (1997): "Consumption Choice and Asset Pricing with a Non-price-taking Agent," Economic Theory, 10(3), 437-462.

Bhattacharya, U., and M. Spiegel (1991): "Insiders, Outsiders, and Market Breakdowns," The Review of Financial Studies, 4(2), 255-282.

Brav, A., G. M. Constantinides, and C. C. Geczy (2002): "Asset Pricing with Heterogeneous Consumers and Limited Participation: Empirical Evidence," Journal of Political Economy, 110(4), 793-824.

Brunnermeier, M., And L. H. Pedersen (2005): "Predatory Trading," Journal of Finance, 60(4), 1825-1863.

Brunnermeier, M. K., and L. Pedersen (2009): "Market Liquidity and Funding Liquidity," Review of Financial Studies, 22, 2201-2238.

Carvajal, A., M. Rostek, and M. Weretka (2012): "Competition in Financial Innovation," Econometrica, 80(5), 1895-1936.

Carvajal, A., and M. WeretKa (2012): "No-arbitrage, state prices and trade in thin financial markets," Economic Theory, 50(1), 223-268. 
Chien, Y., And H. Lustig (2010): "The Market Price of Aggregate Risk and the Wealth Distribution," The Review of Financial Studies, 23(4), 1596-1650.

Constantinides, G. M., And D. Duffie (1996): "Asset Pricing with Heterogeneous Consumers," Journal of Political Economy, 104(2), 219-240.

Corbae, D., and R. Levine (2018): "Competition, Stability, and Efficiency in Financial Markets," Working Paper.

Craig, B., ANd Y. Ma (2018): "Intermediation in the Interbank Lending Market," mimeo.

De Loecker, J., J. Eeckout, and G. Unger (2018): "The Rise of Market Power and the Macroeconomic Implications," mimeo.

Denbee, E., C. Julliard, Y. Li, and K. Yuan (2018): "Network Risk and Key Players: A Structural Analysis of Interbank Liquidity," mimeo.

Donaldson, J. R., and G. Piacentino (2017): "Netting," mimeo.

Donaldson, R. G. (1992): "Costly Liquidation, Interbank Trade, Bank Runs and Panics," Journal of Financial Intermediation, 1(1), 59-82.

EisenberG, L., And T. H. Noe (2001): "Systemic Risk in Financial Systems," Management Science, 47(2), 205-336.

Elliott, M., B. Golub, and M. O. Jackson (2014): "Financial Networks and Contagion," American Economic Review, 104(10), 3115-53.

Erol, S. (2018): "Network Hazard and Bailouts," mimeo.

FARBOodi, M. (2016): "Intermediation and Voluntary Exposure to Counterparty Risk," mimeo.

Foster, F. D., And S. Viswanathan (1996): "Strategic Strading When Agents Forecast the Forecasts of Others," The Journal of Finance, 51(4), 1437-1478.

GLeBkin, S. (2017): "Strategic Trading Without Normality," mimeo.

Gromb, D., and D. Vayanos (2002): "Equilibrium and Welfare in Markets with Financially Constrained Arbitrageurs," Journal of Financial Economics, 66, 361-407. 
Gutierrez, G., And T. Philippon (2016): "Investment-less Growth: An Empirical Investigation," NBER Working Paper $2289 \%$.

Hau, H., P. Hoffmann, S. Langfield, and Y. Timmer (2017): "Discriminatory Pricing of Over-the-Counter Derivatives," Swiss Finance Institute Research Paper No. 17-70.

Hellwig, C., And G. Lorenzoni (2009): "Bubbles and Self-Enforcing Debt," Econometrica, 77(4), 1137-1164.

Kehoe, T. J., And D. K. Levine (1993): "Debt-Constrained Asset Markets," The Review of Economic Studies, 60(4), 865-888.

Kocherlakota, N. R. (1996): "Implications of Efficient Risk Sharing without Commitment," The Review of Economic Studies, 63(4), 595-609.

KoiJen, R. S., And M. Yogo (forthcoming): "A Demand System Approach to Asset Pricing," Journal of Political Economy.

Kyle, A. S. (1985): "Continuous Auctions and Insider Trading," Econometrica, 54(6), $1315-1335$.

(1989): "Informed Speculation with Imperfect Competition," The Review of Economic Studies, 56(3), 317-355.

Leitner, Y. (2005): "Financial Networks: Contagion, Commitment, and Private Sector Bailouts," The Journal of Finance, 60(6), 2925-2952.

Malamud, S., And A. Teguia (2017): "Asset Pricing with Large Investors," mimeo SFI Ecole Polytechnique Federale de Lausanne, and CEPR.

PRITSkeR, M. (2005): "Large Investors: Implications for Large Investors: Implications for Equilibrium Asset Returns, Shock Absorption, and Liquidity," Finance and Economics Discussion Series (FEDS) Working Paper No. 2005-36.

Rochet, J.-C., And J. Tirole (1996): "Interbank Lending and Systemic Risk," Journal of Money, Credit and Banking, 28(4), 733-762.

Rostek, M., And M. Weretka (2012): "Price Inference in Small Markets," Econometrica, 80(2), 687-711. 
_ (2015): "Dynamic Thin Markets," The Review of Financial Studies, 28(10), 29462992.

Roukny, T., S. Battiston, And J. E. Stiglitz (2018): "Interconnectedness as a source of uncertainty in systemic risk," Journal of Financial Stability, 35, 93-106.

Vayanos, D. (1999): "Strategic Trading and Welfare in a Dynamic Market," The Review of Economic Studies, 66(2), 219-254.

- (2001): "Strategic Trading in a Dynamic Noisy Market," The Journal of Finance, 56(1), 131-171.

Weretka, M. (2011): "Endogenous Market Power," Journal of Economic Theory, 146(6), $2281-2306$.

Xiong, W. (2001): "Convergence trading with wealth effects: an amplification mechanism in financial markets," Journal of Financial Economics, 62(2), 247-292.

Zawadowski, A. (2013): "Entangled Financial Systems," The Review of Financial Studies, 26(5), 1291-1323. 


\section{Appendix A: Proofs of Propositions}

Proof of Proposition 1:

Similar in spirit to the analysis of Eisenberg and Noe (2001), we do not take the decision of agents to repay as given. Let $\delta_{i}(z)$ is an indicator function for whether agent $i$ repays its obligations at $t=2$, and takes value $\delta_{i}(z)=1$ if agent $j$ repays. If agent $i$ does not issue a state-contingent claim for state $z$, we trivially characterize it as not defaulting, or $\delta_{i}(z)=1$. To proceed with the analysis, we first rewrite and stack the vector of no default conditions $c_{i 2}^{N D}(z) \geq c_{i 2}^{D}(z)$ into a matrix equation for all agents:

$$
\xi(Y(z) \vec{k}+B(z) D(z) \vec{\delta}(z)) \geq \vec{\alpha}(z)-\xi R_{f} \vec{s}
$$

where $Y(z)$ is a nonnegative diagonal matrix with entries $Y_{i i}(z)=y_{i}(z), \vec{k}$ is the vector of agent capital, $A(z)$ is a nonnegative matrix that has entries $A_{i j}(z)=-a_{i}(j, z) / a_{j}(j, z)$ with $A_{i i}(z)=0, \vec{\alpha}(z)$ has entries $\vec{\alpha}(z)=-a_{i}(i, z)$ and $B(z)=-\operatorname{diag}\left(a_{j}(j, z)\right)$. Implicitly, the outside investor has index $N+1$ with entries $A_{N+1 j}(z)=-D(j, z) / a_{j}(j, z)$. The above condition states that the benefit of default, the RHS, which is the forgeone repayments of obligations and storage, must be (weakly) less than the cost, the LHS, which is the loss in revenue from production and the repayments of claims of other agents from distress.

Let us call $\vec{\delta}(z)$, the stacked indicators of whether agent $i$ repays, a repayment vector. We can then rewrite the above inequality condition for the default decisions of agents as a fixed-point problem:

$$
\vec{\delta}(z)=\mathbf{1}\{Y(z) \vec{k}+A(z) B(z) \vec{\delta}(z)-\vec{g}(z) \geq 0\}
$$

where:

$$
\vec{g}(z)=\xi^{-1} B(z) \mathbf{1}-R_{f} \vec{s}
$$

is fixed at $t=1$, as are $\vec{k}$ and $A(z)$. The LHS is nonnegative by construction (with a range of $\{0,1\}^{N}$ ) and (weakly) monotonically increasing in the repayment vector $\vec{\delta}(z)$ element-byelement. since $A(z) \vec{\delta}(z)$ is (weakly) monotonically increasing in $\vec{\delta}(z)$, element-by-element.

In what follows, we will make use of the following lemma. 20

\footnotetext{
${ }^{20}$ In the proof the Lemma, we modify the default and consumption vectors to include agent $N+1$ as the last entry, and set $\delta_{N+1}(z)=1 \forall z$ trivially. Having the outside investor as part of $B(z)$, however, ensures
} 
Lemma 1: Define:

$$
f(\vec{\delta}(z), z)=\mathbf{1}\{Y(z) \vec{k}+A(z) B(z) \vec{\delta}(z)-\vec{g}(z) \geq 0\}
$$

then $f(\vec{\delta}(z), z)$ has a largest and a smallest fixed point, $\vec{\delta}^{*}(z)$ and $\vec{\delta}_{*}(z)$, respectively, for every $z$, such that $\vec{\delta}^{*}(z) \geq \vec{\delta}_{*}(z)$ element-by-element.

The claim then follows from arguments similar to those in Eisenberg and Noe (2001) Lemma 5 and Milgrom and Roberts (1994) Theorem 3. We can treat $f(\vec{\delta}(z), z)$ as an interative map:

$$
\vec{\delta}^{n}(z)=f\left(\vec{\delta}^{n-1}(z), z\right)=\mathbf{1}\left\{Y(z) \vec{k}+A B \vec{\delta}^{n-1}(z)-\vec{g} \geq 0\right\}
$$

is (weakly) increasing in $Y(z) \vec{k}$ and decreasing in $\vec{g}$, and therefore increasing in $\vec{s}$ by the definition of $\vec{g}$. Since $f\left(\vec{\delta}^{n-1}(z), z\right)$ is nondecreasing in the operator $\vec{\delta}^{n-1}(z)$ and, by the Monotone Convergence Theorem, converges pointwise from below to $\vec{\delta}(z)$, it follows that $\vec{\delta}(z)$ is (weakly) increasing in $Y(z) \vec{k}$ and $\vec{s}$, (element-by-element). This holds for each fixed point $\vec{\delta}^{* *}(z)$ of $f(\vec{\delta}(z), z)$.

As the repayment vector represents the incentive compatibility constraints of agents at $t=1$, it follows that all of these constraints are weakly relaxed in $Y(z) \vec{k}$ and $\vec{s}$.

\section{Proof of Lemma 1:}

We establish several properties of $f(\vec{\delta}(z), z)$. It is straightforward from the above arguments that $f(\vec{\delta}(z), z)$ is nonnegative, (weakly) monotonically increasing in $\vec{\delta}(z)$, and non-expansive in the $L^{1}$-norm. To see that $f(\vec{\delta}(z))$ is non-expansive in the $L^{1}$-norm, we recognize that:

$$
\begin{aligned}
& \left\|f(\vec{\delta}(z), z)-f\left(\vec{\delta}^{+}(z), z\right)\right\| \\
& =\left\|\mathbf{1}\{A(z) B(z) \vec{\delta}(z) \geq \vec{g}(z)-Y(z) \vec{k}\}-\mathbf{1}\left\{A(z) B(z) \vec{\delta}^{+}(z) \geq \vec{g}(z)-Y(z) \vec{k}\right\}\right\| \\
& =\mathbf{1}\{A(z) B(z) \vec{\delta}(z) \geq \vec{g}(z)-Y(z) \vec{k}\}^{\prime} \mathbf{1}\left\{A(z) B(z) \vec{\delta}^{+}(z) \leq \vec{g}(z)-Y(z) \vec{k}\right\} \\
& +\mathbf{1}\{A(z) B(z) \vec{\delta}(z) \leq \vec{g}(z)-Y(z) \vec{k}\}^{\prime} \mathbf{1}\left\{A(z) B(z) \vec{\delta}^{+}(z) \geq \vec{g}(z)-Y(z) \vec{k}\right\}
\end{aligned}
$$

that the columns of $B(z)$ sum to 1 . For Lemma 1, the outside investor weakly consumes more when less agents default, consistent with the Pareto ranking of consumption vectors. 
where $\|\cdot\|$ is the $L^{1}$-norm. Since $\left\|f(\vec{\delta}(z), z)-f\left(\vec{\delta}^{+}(z), z\right)\right\|$ is the sum of the elements $i$ for which $f_{i}(\vec{\delta}(z), z) \neq f_{i}\left(\vec{\delta}^{+}(z), z\right)$, this difference can be no greater than the sum of the elements for which $A(z) B(z) \vec{\delta}(z)-\vec{g}(z)+Y(z) \vec{k}$ and $A(z) B(z) \vec{\delta}^{+}(z)-\vec{g}(z)+Y(z) \vec{k}$ differ, or:

$$
\begin{aligned}
\left\|f(\vec{\delta}(z), z)-f\left(\vec{\delta}^{+}(z), z\right)\right\| & \leq \iota^{\prime} \mathbf{1}\left\{\left|A(z) B(z) \vec{\delta}^{+}(z)-A(z) B(z) \vec{\delta}(z)\right| \geq 0\right\} \\
& =\iota^{\prime} \mathbf{1}\left\{\left|A(z) B(z)\left(\vec{\delta}^{+}(z)-\vec{\delta}(z)\right)\right| \geq 0\right\} .
\end{aligned}
$$

Since the sum of the columns of $A(z)$ is less than or equal to 1 by construction, it follows that $\|A(z)\| \leq 1$, and consequently that:

$$
\left\|A(z) B(z)\left(\vec{\delta}^{+}(z)-\vec{\delta}(z)\right)\right\| \leq\left\|B(z)\left(\vec{\delta}^{+}(z)-\vec{\delta}(z)\right)\right\|=\vec{d}(z)^{\prime}\left\|\vec{\delta}^{+}(z)-\vec{\delta}(z)\right\|
$$

with $\vec{d}>>0$ having only nonnegative entries. Consequently, the sum of the elements of $\left|A(z) B(z)\left(\vec{\delta}^{+}(z)-\vec{\delta}(z)\right)\right|$ is no greater than the sum of the elements of $\left|B(z)\left(\vec{\delta}^{+}(z)-\vec{\delta}(z)\right)\right|$. Since $1\left\{\left|A(z) B(z)\left(\vec{\delta}^{+}(z)-\vec{\delta}(z)\right)\right| \geq 0\right\}$ and $\mathbf{1}\left\{\left|B(z)\left(\vec{\delta}^{+}(z)-\vec{\delta}(z)\right)\right| \geq 0\right\}$ only require elements to be nonnegative to contribute to the $L^{1}$-norm, it follows that:

$$
\begin{aligned}
\left\|f(\vec{\delta}(z), z)-f\left(\vec{\delta}^{+}(z), z\right)\right\| & \leq \iota^{\prime} \mathbf{1}\left\{\left|A(z) B(z)\left(\vec{\delta}^{+}(z)-\vec{\delta}(z)\right)\right| \geq 0\right\} \\
& \leq \iota^{\prime} \mathbf{1}\left\{\left|B(z)\left(\vec{\delta}^{+}(z)-\vec{\delta}(z)\right)\right| \geq 0\right\} \\
& =\iota^{\prime} \mathbf{1}\left\{\left|\vec{\delta}^{+}(z)-\vec{\delta}(z)\right| \geq 0\right\} \\
& =\left\|\vec{\delta}^{+}(z)-\vec{\delta}(z)\right\|,
\end{aligned}
$$

where $\mathbf{1}\left\{\left|B(z)\left(\vec{\delta}^{+}(z)-\vec{\delta}(z)\right)\right| \geq 0\right\}=\mathbf{1}\left\{\left|\vec{\delta}^{+}(z)-\vec{\delta}(z)\right| \geq 0\right\}$ follows since $B(z)$ are all nonnegative, which establishes the claim. Furthermore, $(S, \leq)$ defines a complete lattice, where $S=\{0,1\}^{N}$. Consequently, we can apply the Knaster-Tarski Fixed Point Theorem for complete lattices to conclude that $f(\vec{\delta}(z), z)$ has a largest and a smallest fixed point, $\delta^{*}(z)$ and $\delta_{*}(z)$, respectively, for every $z$.

If a column of $A(z)$ is populated by zeros (no borrowing by an agent) or a row is populated by zeros (no lending by an agent), then we can determine whether the agent will default independent of the rest of the network. Let this agent be $i$. With no borrowing, agent $i$ never defaults since default is costly, and $\delta_{i}(z)=1$. With no lending, default is determined 
as $\delta_{i}(z)=1\left\{y_{i}(z) k_{i}-c_{i 1} \geq 0\right\}$. In both situations, we can set $\delta_{i}(z)$ to its value, remove the row from $A(z)$, and move the column into $\vec{c}_{1}$. The remaining matrix $\tilde{A}(z)$ will have at least one nonzero entry in every row and column.

\section{Proof of Corollary 1:}

From the proof of Proposition $1, \vec{\delta}(z)$ is (weakly) increasing in $Y(z) z \vec{k}+\vec{s}$. Define $x=Y(z) z \vec{k}+\vec{s}$ and $\frac{\partial \vec{\delta}(z)}{\partial x}$ to be the Jacobian matrix that measures the marginal relaxation of the agents' I.C. constraints. It follows that $\frac{\partial \vec{\delta}(z)}{\partial s_{i}}=R_{f} \frac{\partial \vec{\delta}(z)}{\partial x} e_{i}$, where $e_{i}$ is the Euclidian basis vector in direction $i$. Similarly, $\frac{\partial \vec{\delta}(z)}{\partial k_{i}}=y_{i}(z) \frac{\partial \vec{\delta}(z)}{\partial x} e_{i}$. Consequently, $\frac{\partial \vec{\delta}(z)}{\partial k_{i}}=\frac{y_{i}(z)}{R_{f}} \frac{\partial \vec{\delta}(z)}{\partial s_{i}}$. It then follows that agent $i^{\prime} s$ I.C. constraint relaxes by $\frac{y_{i}(z)}{R_{f}}$ in state $z$ if agent $i$ substitutes savings for its risky production.

\section{Proof of Proposition 2:}

Since no agents default in equilibrium, the shadow value of an increase in debt issuance by agent $i$ today is absorbed by the appropriate Lagrange multiplier, which increases the asset price despite the lack of change in agent $j^{\prime} s$ marginal utility by Kuhn-Tucker. FONCs are then valid.

Let us normalize the Lagrange multiplier on wealth be $\varphi_{i}$ for (5) to be $\varphi_{i} e_{i}^{\gamma}$. The FONCs for $c_{i 1}, k_{i}, s_{i}, d_{i}$, and $\vec{b}_{i}$ in problem (5) are given by:

$$
\begin{aligned}
c_{i 1}: & u^{\prime}\left(c_{i 1}\right)-\varphi_{i} e_{i}^{\gamma} \leq 0\left(=\text { if } c_{i 1}>0\right), \\
k_{i}: & \sum_{z \in \mathcal{Z}} \pi(z) u_{2}^{\prime}\left(c_{i 2}(z)\right) y_{i}(z)-\varphi_{i} e_{i}^{\gamma}+\xi e_{i}^{\gamma} \sum_{z \in \mathcal{Z}} \pi(z) \psi_{i}(z) y_{i}(z) \leq 0\left(=\text { if } k_{i}>0\right), \\
s_{i}: & \sum_{z \in \mathcal{Z}} \pi(z) u_{2}^{\prime}\left(c_{i 2}(z)\right) R_{f}-\varphi_{i} e_{i}^{\gamma}+R_{f} \xi e_{i}^{\gamma} \sum_{z \in \mathcal{Z}} \pi(z) \psi_{i}(z) \leq 0\left(=\text { if } s_{i}>0\right), \\
a_{i}(i, z): & -\pi(z) u_{2}^{\prime}\left(c_{i 2}(z)\right)+\varphi_{i} e_{i}^{\gamma}\left(q_{i}(z)+\frac{\partial q_{i}(z)}{\partial a_{i}(i, z)} a_{i}(i, z)\right)-\pi(z) \psi_{i}(z) e_{i}^{\gamma} \\
& \leq 0\left(=i f a_{i}(i, z)>0\right), \\
\vec{a}_{-i}^{\prime}(z): & L_{i}\left(\pi(z) u_{2}^{\prime}\left(c_{i 2}(z)\right) \iota-\varphi_{i} e_{i}^{\gamma}\left(\vec{q}(z)+\frac{\partial \vec{q}(z)}{\partial \vec{a}_{-i}} \odot \vec{a}_{-i}^{\prime} \odot \vec{l}_{i}\right)+\pi(z) \psi_{i}(z) e_{i}^{\gamma} \xi \vec{l}_{i}\right) \\
\leq & \overrightarrow{0}\left(=i f \vec{a}_{-i}>0\right),
\end{aligned}
$$

where $L_{i}$ selects the rows that are not $i$. Therefore, the above represent the FONCs for agent $i^{\prime} s$ problem. Since $u_{1}(\cdot)$ satisfies the Inada condition, $c_{i 1}>0$ and the first FOC binds with equality. 
Define $h_{i}=\frac{e_{i}}{\sum_{i=1}^{N} e_{i}}$ to be the effective share of scaled equity of agent $i$. Let us conjecture that each agent's optimal policies are such that, for policy $s_{i}$, we can decompose $s_{i}$ into $s_{i}=\hat{s}_{i} e_{i}$, where $\hat{s}_{i}$ is independent of $e_{i}$ and the level of equity of any other agent. From equation (11), we can rewrite the stacked incentive compatibility conditions as:

$$
\iota=\mathbf{1}\{Y(z) \overrightarrow{\hat{k}}+B(z) \hat{D}(z) \iota-\overrightarrow{\hat{g}}(z) \geq 0\}
$$

where:

$$
\overrightarrow{\hat{g}}(z)=\xi^{-1} \hat{B}(z) \iota-R_{f} \overrightarrow{\hat{s}}
$$

which depends on $e_{i}$ only through $h_{i} \forall i$. Consequently, the default decision satisfies the conjectured decomposition.

Furthermore, imposing market-clearing we can express the price of agent $i^{\prime} s$ debt referencing state $z$ as: $D(i, z)=f_{i}\left(q_{i}(z), z\right) \sum_{j=1}^{N} e_{j}$

$$
q_{i}(z)=f_{i}^{-1}\left(\frac{-\sum_{j=1}^{N} a_{j}(i, z)}{\sum_{j=1}^{N} e_{j}}, z\right)=f_{i}^{-1}\left(-\sum_{j=1}^{N} h_{j} \hat{a}_{j}(i, z), z\right) .
$$

By the Inverse Function Theorem, the inverse of $f_{i}\left(q_{i}(z), z\right)$ exists (locally) wherever $\frac{\partial f_{i}(x, z)}{\partial x} \neq$ 0. Further, $\frac{\partial f_{i}^{-1}(x, z)}{\partial x}=\left(\frac{\partial f_{i}(x, z)}{\partial x}\right)^{-1}<0$. Consequently, it follows that $\frac{\partial q_{j}(z)}{\partial \hat{a}_{j}(i, z)}>0 \forall(i, z)$, with the inequalities strict since $f(\cdot, z)$ is a strictly increasing function.

Since utility is homogeneous in degree $e_{i}^{\gamma}$, we can then rewrite the FONCs as:

$$
\begin{aligned}
\hat{c}_{i 1} & : \quad u_{1}^{\prime}\left(\hat{c}_{i 1}\right)-\varphi_{i}=0 \\
\hat{k}_{i} & : \sum_{z \in \mathcal{Z}} \pi(z) \frac{u_{2}^{\prime}\left(\hat{c}_{i 2}(z)\right)}{u_{1}^{\prime}\left(\hat{c}_{i 1}\right)} y_{i}(z)+\xi \sum_{z \in \mathcal{Z}} \pi(z) \frac{\psi_{i}(z)}{u_{1}^{\prime}\left(\hat{c}_{i 1}\right)} y_{i}(z) \leq 1\left(=\text { if } \hat{k}_{i}>0\right), \\
\hat{s}_{i}: & \sum_{z \in \mathcal{Z}} \pi(z) \frac{u_{2}^{\prime}\left(\hat{c}_{i 2}(z)\right)}{u_{1}^{\prime}\left(\hat{c}_{i 1}\right)} R_{f}+R_{f} \xi \sum_{z \in \mathcal{Z}} \pi(z) \frac{\psi_{i}(z)}{u_{1}^{\prime}\left(\hat{c}_{i 1}\right)} \leq 1\left(=\text { if } \hat{s}_{i}>0\right), \\
\hat{a}_{i}(i, z): & -\pi(z) \frac{u_{2}^{\prime}\left(\hat{c}_{i 2}(z)\right)}{u_{1}^{\prime}\left(\hat{c}_{i 1}\right)}+q_{i}(z)-h_{i} \frac{\partial q_{i}(z)}{\partial \hat{a}_{i}(i, z)} \hat{a}_{i}(i, z)-\pi(z) \frac{\psi_{i}(z)}{u_{1}^{\prime}\left(\hat{c}_{i 1}\right)} \leq 0\left(=i f \hat{a}_{i}(i, z)<0\right), \\
\overrightarrow{\hat{a}}_{-i}^{\prime}(z): & \pi(z) \frac{u_{2}^{\prime}\left(\hat{c}_{i 2}(z)\right)}{u_{1}^{\prime}\left(\hat{c}_{i 1}\right)} \iota-\vec{q}(z)-h_{i} \frac{\partial \vec{q}(z)}{\partial \overrightarrow{\hat{a}}_{-i}(z)} \odot \overrightarrow{\hat{a}}_{-i}^{\prime}(z)+\pi(z) \frac{\psi_{i}(z)}{u_{1}^{\prime}\left(\hat{c}_{i 1}\right)} \xi \iota \\
\leq & \overrightarrow{0}\left(=i f \overrightarrow{\hat{a}}_{-i}(z)>0\right),
\end{aligned}
$$


and by definition of the budget constraint at $t=2$ :

$$
\hat{c}_{i}(z)=y_{i}(z) \hat{k}_{i}+\sum_{j \neq i} \hat{a}_{i}(j, z)+R_{f} \hat{s}_{i}+\hat{a}_{i}(i, z)
$$

Finally, the budget constraint at $t=1$, which will hold with equality by efficiency, can be expressed as:

$$
\hat{c}_{i 1}+\hat{k}_{i}+\sum_{z \in \mathcal{Z}} \overrightarrow{\hat{a}}_{-i}(z) \vec{q}(z)+s_{i}=1-\sum_{z \in \mathcal{Z}} q_{i}(z) \hat{a}_{i}(i, z) .
$$

The rewritten FONCs are independent of $e_{i} \forall i \in\{1, \ldots, N\}$, and, defining $\Lambda_{i}(z)=\pi(z) \frac{u_{2}^{\prime}\left(\hat{c}_{i 2}(z)\right)+\xi \psi_{i}(z)}{u_{1}^{\prime}\left(\hat{c}_{i 1}\right)}$ to be the effective state price of agent $i$ in state $z$, confirm our conjecture.

It then follows that the indirect utility of agent $i$ from its trading and repayment game is:

$$
U_{1}^{i}\left(e_{i}, \vec{e}_{-i}\right)=e_{i}^{1-\gamma} u_{1}\left(\hat{c}_{i 1}\right)+e_{i}^{1-\gamma} \sum_{z \in \mathcal{Z}} \pi(z) u_{2}\left(\hat{c}_{i 2}(z)\right)=\tilde{U}_{1}^{i}\left(h_{i}, \vec{h}_{-i}\right) e_{i}^{1-\gamma} .
$$

To see that all the controls, normalized by the equity of agent $i e_{i}$ are bounded, we consider the cases in which agent $i$ does and does not issue state-contingent claims. When agent $i$ does not issue any state-contingent claims, then by the balance-sheet constraint, it can only self-finance its uses of funds, and therefore $\hat{c}_{i 1},-\hat{a}_{i}(i, z), \hat{k}_{i}, \hat{a}_{j}(i, z) \leq 1$. When agent $i$ issues state-contingent claims, then the total normalized resources of all agents in the network is $N$ plus borrowing from the outside creditor, agent 0 .

By assumption, the credit that can be extracted from the outside investor $N+1$ in any state, $q_{i}(z) f_{i}\left(q_{i}(z), z\right) \sum_{j} e_{j}$, is bounded from above. It then follows that:

$$
-\hat{a}_{i}(i, z) \leq\left(1+\sup _{q_{i}(z) b_{N+1 i}(z)} q_{i}(z) f_{i}\left(q_{i}(z), z\right)\right) \sum_{j \neq i} e_{j}
$$

which is bounded, and can be rewritten as:

$$
\begin{aligned}
-\hat{a}_{i}(i, z) \leq & \frac{1}{h_{i}}\left(1+\sup _{q_{i}(z) b_{N+1 i}(z)} q_{i}(z) f_{i}\left(q_{i}(z), z\right)\right) \leq \frac{1}{\min \left\{h_{j}\right\}}\left(1+\sup _{q_{i}(z) b_{N+1 i}(z)} q_{i}(z) f_{i}\left(q_{i}(z), z\right)\right) \\
& =-\widehat{\bar{a}}_{i}(i, z) .
\end{aligned}
$$

Since borrowing in each state is bounded from above, total borrowing by agent $i$ is bounded from above.

Therefore, $\hat{a}_{i}(i, z) \geq \widehat{\bar{a}}_{i}(i, z)$, and $\hat{c}_{i 1}, \hat{k}_{i}, \hat{a}_{i}(j, z) \leq 1-\widehat{\bar{a}}_{i}(i, z)$. The controls are trivially 
bounded from below by 0 because of the investment constraints on agent $i^{\prime} s$ problem. Since the controls lie in a closed and bounded set, they lie in a compact set by the Heine-Borel Theorem.

Define $\mathcal{X}_{i}$ to be the state space at date $t$ for agent $i \mathcal{X}_{i}=\mathbb{R}_{+}^{N} \times \mathcal{S}_{i}^{N \times|\mathcal{Z}|} \times \Sigma(-i)$, where $\Sigma(-i)$ is the space of strategies of the other agents in the network. Notice that, given the homotheticity of agent utility, it follows that $U_{0}^{i}\left(e_{i}, \vec{e}_{-i}\right)=U_{0}^{i}\left(h_{i}, \vec{h}_{-i}\right) e_{i}^{1-\gamma}$, where $h_{j} \in[0,1] \forall j \in\{1, \ldots, N\}$, and the range of the controls $\hat{k}_{i}, \hat{s}_{i}, \hat{a}_{i}(i, z)$, and $\hat{a}_{i}(j, z)$ lies in a compact, convex set. As such, the set of admissible controls normalized by equity $\mathcal{M} \subseteq \mathbb{R}_{+}^{N \times|\mathcal{Z}|+2}$ is compact and convex, where $g_{i} \in \mathcal{M}$ is a $N \times|\mathcal{Z}|+2$ tuple $g_{i}=\left[\begin{array}{llllllll}\hat{c}_{i 1} & \hat{k}_{i} & \hat{a}_{i}\left(i, z_{1}\right) & \ldots & \hat{a}_{i}\left(z_{|\mathcal{Z}|}\right) & \overrightarrow{\hat{a}}_{-i}\left(z_{1}\right) & \ldots & \overrightarrow{\hat{a}}_{-i}\left(z_{|\mathcal{Z}|}\right)\end{array}\right]^{\prime}$, which maps to the controls in the original problem as $g_{i} e_{i}$. Then $G_{i}: \mathcal{X}_{i} \rightarrow \mathcal{M}$ is a compact-valued correspondence.

Since the objective $u_{2}\left(c_{i 2}(z)\right)$ is continuous in the states, and consequently in the controls, and the correspondence $G_{i}$ is compact-valued, then by Berges' Theory of the Maximum there exists a solution to the agent's problem and the optimal $G_{i}^{*}$ is an upper-hemicontinuous correspondence. Importantly, it is the homogeneity of the optimal controls with respect to the equity of agent $i e_{i}$ that allows us to express the space of controls as a compact-valued correspondence to satisfy the theorem.

Given that $G_{i}^{*}$ is an upper-hemicontinuous correspondence $\forall i \in\{1, \ldots, N\}$, applying Kakutani's Fixed Point Theorem on the market-clearing conditions for agent debt establishes existence of a fixed point that is an equilibrium.

\section{Proof of Corollary 2:}

Consider the autarkic equilibrium in which $a_{i}(i, z)=0$ and $a_{i}(j, z)=0 \forall i \in\{1, . ., N\}$ and $z \in \mathcal{Z}$. Locally, if agent $i$ instead wishes to deviate from this equilibrium and issue a total of $\varepsilon$ of state-contingent claims across states, then it will never default since the benefit of repaying at $t=2$ is $\varepsilon$ while the cost is $(1-\xi)\left(y_{i}(z) k_{i}+s_{i}\right)$ for $\varepsilon$ small enough. Consequently, $\delta_{i}(z) \equiv 1$. We now check that $a_{i}(j, z)=a_{j}(i, z)=0 \forall j$ is a best response. It is then the case that no agents defaults either, or $\vec{\delta}(z) \equiv \overrightarrow{1}_{N \times 1}$.

Rewriting the FONCs for agent $i$ for issuing normalized claim $-\hat{a}_{i}(i, z)$ and purchasing 
the claim $-\hat{a}_{j}(j, z)$ of agent $j$ are:

$$
\begin{aligned}
& \hat{a}_{i}(i, z): q_{i}(z) \leq \pi(z) \frac{u_{2}^{\prime}\left(\hat{c}_{i 2}(z)\right)}{u_{1}^{\prime}\left(\hat{c}_{i 1}\right)}-h_{i} \frac{\partial q_{i}(z)}{\partial \hat{a}_{i}(i, z)} \hat{a}_{i}(i, z) \quad\left(=\text { if } \hat{a}_{i}(i, z)<0\right), \\
& \hat{a}_{i}(j, z): q_{j}(z) \geq \pi(z) \frac{u_{2}^{\prime}\left(\hat{c}_{i 2}(z)\right)}{u_{1}^{\prime}\left(\hat{c}_{i 1}\right)}-h_{i} \frac{\partial q_{j}(z)}{\partial \hat{a}_{i}(j, z)} \hat{a}_{i}(j, z) \quad\left(=\text { if } \hat{a}_{i}(j, z)>0\right) .
\end{aligned}
$$

Similar to Alvarez and Jermann (2000), at the autarky allocations, there is a wedge in state prices between agent $i$ and $j$ at the autarky allocation $\left\{c_{i 1}^{\text {aut }}, c_{i 2}^{\text {aut }}(z)\right\}$. Now set:

$$
q_{i}(z)=\bar{q}(z)=\max _{j \in\{1, \ldots, N\}} \pi(z) \frac{u_{2}^{\prime}\left(c_{j 2}^{\text {aut }}(z)\right)}{u_{1}^{\prime}\left(c_{j 1}^{a u t}\right)} \forall i \in\{1, \ldots, N\} .
$$

Then, $q_{i}(z) \geq \pi(z) \frac{u_{2}^{\prime}\left(\hat{c}_{i 2}^{\text {aut }}(z)\right)}{u_{1}^{\prime}\left(\hat{c}_{i 1}^{\text {aut }}\right)}$ and $q_{i}(z) \geq \pi(z) \frac{u_{2}^{\prime}\left(\hat{c}_{j 2}^{\text {aut }}(z)\right)}{u_{1}^{\prime}\left(\hat{c}_{j 1}^{a u t}\right)}$ from the perspectives of agents $i$ and $j$, respectively, if either were to trade security $q_{i}(z)$. It must then be the case that both would want to short-sell the security, and similarly with any other pair of agents, as there is a wedge in their marginal utilities and that of the agent with the highest marginal rate of transformation. Consequently, all agents have incentive to issue securities to each other at the autarkic allocation, but none want to buy.

We now have to consider trading with the outside investor, investor $N+1$. At zero issuance, $q_{i}(z)=f_{i}^{-1}(0, z)$. Suppose that $f_{i}^{-1}(0, z)=\bar{q}(z)$ at zero issuance. It then follows that there are no gains-from-trade between agent $i$ and the outside investor, as the outside investor has zero demand while agent $i$ wishes to short-sell. The converse is straightforward from the observation that, if the allocation is autarky, then $f_{i}^{-1}(0, z)=\bar{q}(z) \forall(i, z)$ or else agent $i$ would have incentive to trade away from autkary, violating the assumption that autarky is an equilibrium.

Consequently, autarky is a supported allocation in the trading game if $f_{i}^{-1}(0, z)=$ $\bar{q}(z) \forall(i, z)$.

\section{Proof of Proposition 3:}

Consider the Nash Equilibrium in demand schedules allocation in the economy with imperfect competition, $\left\{\left(c_{i 1}, k_{i}, s_{i},\left\{c_{i}(z)\right\}_{z \in \mathcal{Z}}\right)\right\}_{i=1}^{N}$. Define

$$
\Lambda_{i}(z)=q(j, z)+\frac{\partial q(j, z)}{\partial a_{i}(j, z)} a_{i}(i, z) \forall(i, j, z)
$$


to be the implied state price deflator of agent $i$ in state $z$. Notice the implied state price deflator can include a collateral premium.

Now consider a fictitious incomplete markets economy in which all agents are instead competitive. We will try to derive the implied market structure, indexed by a set of $M$ securities with arbitrary $M \times K$ payoff matrix $D$. Since there are $K$ possible states of the world, it follows that we need at most $K$ linearly independent securities for markets to be complete, and consequently $M \leq K$. If markets are incomplete, then these $M$ securities are rank deficient, and consequently specifying at most $K$ potentially tradable securities is without loss. Since assets cannot distinguish between some (subset of) states, the marketimplied asset span spans $M$ (linear combinations of the $K$ ) states.

Let the price vector for these $M$ assets be $\vec{p}$ and define $\frac{D}{p}$ to be the dividend yield matrix with elements $\frac{d_{i j}}{p_{i}}$, where $d_{i j}$ is the payoff of the $i^{t h}$ security in state $j$, and $q_{i}$ is the price of security $i$. We follow the convention that, since by no arbitrage $p_{i}=0$ iff $d_{i j}=0$ $\forall j \in\{1, \ldots, M\}$, that $\frac{d_{i j}}{p_{i}}=0$ in this contingency.

No arbitrage then requires that

$$
\mathcal{M} \frac{D}{p}=\iota_{N} \iota_{M}^{\prime}
$$

where $\mathcal{M}$ is the $N \times K$ matrix with elements $\mathcal{M}_{i j}=\Lambda_{i}(j)$ for intermediary $i$ and state $j$, and $\iota_{M}$ is the $M \times 1$ vector of one.

For fixed $M$, this equation can be rewritten as a matrix equation:

$$
\left(I_{M} \otimes \mathcal{M}\right) \operatorname{vec}\left(\frac{D}{p}\right)=\operatorname{vec}\left(\iota_{N} \iota_{M}^{\prime}\right)
$$

Since $\operatorname{rank}\left(I_{M} \otimes \mathcal{M}\right)=M+\operatorname{rank}(\mathcal{M})$, it follows that there is a unique solution for $\frac{D}{p}$ if $N \geq K$, while the system is under-identified if $N<K$.

As the synthetic assets are potentially derivatives, $\frac{D}{p}$ can have negative entries. We then choose the largest $M$ such that the recovered $\frac{D}{p}$ has rank $M$. We choose the largest $M$ since, if there were an additional asset that replicated the asset span that we ignored, then introducing this additional asset would not initiate trade, since it would already be priced at its no arbitrage value by all intermediaries. Consequently, $M \in \max _{M^{\prime} \in\{0, \ldots, K\}}\left\{\operatorname{rank}\left(\frac{D}{p}\right)=M^{\prime}\right\}$.

With this payoff matrix, we have constructed an incomplete market structure that measures the degree of market incompleteness by replicating the effective asset span of the Nash 
Equilibrium with complete markets. Since at least one state price is misaligned among intermediaries, the rank of $\frac{D}{p}$ must be less than $K$, and markets must be incomplete.

For the second part of the claim, we must also replicate the transfers across agents. Let $X$ be the $N \times K$ matrix with entry $X_{i z}=y_{i}(z) k_{i}-c_{i 1}(z)$ that records the transfers that each agent makes at $t=1$. These transfers have initial cost $X^{\prime} \vec{q}$.

To construct a fictitious incomplete markets, competitive equilibrium that replicates the Nash Equilibrium allocation, we search for a specific $N \times M$ portfolio matrix $A$ that both satisfy agents' Euler Equations and replicates the transfers $X$. To replicate the portfolio transfers, we now also impose that:

$$
X=A D,
$$

from which follows, since $D$ is a $K \times M$ matrix and $M<K$, that

$$
A=X D\left(D^{\prime} D\right)^{-1}
$$

To satisfy the market-clearing condition, it must then be the case that the new portfolios have the same initial costs:

$$
X \vec{q}=A \vec{p},
$$

which, with some manipulation, simplifies to:

$$
\vec{q}=\frac{D}{p}\left(\frac{D^{\prime}}{p} \frac{D}{p}\right)^{-1} \iota
$$

This additional constraint on feasibility imposes that the cost of the portfolios at $t=0$ be the same under the fictitious competitive market structure as in the original.

\section{Proof of Proposition 4 :}

Since there are no cross-holdings, suppose that agent $i$ buys claims from agent $j$ in state $z$. Then, from the FONCs for the respective agents:

$$
\Lambda_{i}(z)-h_{i} \frac{\partial q_{j}(z)}{\partial \hat{a}_{i}(j, z)} \hat{a}_{i}(j, z)=q_{j}(z)=\Lambda_{j}(z)-h_{j} \frac{\partial q_{j}(z)}{\partial \hat{a}_{j}(j, z)} \hat{a}_{j}(j, z),
$$


from which follows that:

$$
\Lambda_{i}(z)-\Lambda_{j}(z)=-h_{j} \frac{\partial q_{j}(z)}{\partial \hat{a}_{j}(j, z)} \hat{a}_{j}(j, z)+h_{i} \frac{\partial q_{j}(z)}{\partial \hat{a}_{i}(j, z)} \hat{a}_{i}(j, z)>0,
$$

and consequently $\Lambda_{i}(z)>\Lambda_{j}(z)$. Consequently, as a result of market power, risk-sharing is imperfect beyond the impairment from the endogenous leverage constraints arising from limited commitment.

Since $\Lambda_{i}(z)>\Lambda_{j}(z)$ in the presence of strategic interaction, it follows that $j$ cannot sell as many claims to $i$ as it would, all else equal, in a competitive environment. By a symmetric argument, $j$ is not able to buy as claims from $i$ as it would in any state $z^{\prime}$ in which it would like to buy, such that $\Lambda_{j}(z)>\Lambda_{i}(z)$, as compared to a competitive environment. Consequently, $j$ is more exposed to the output of its own production than, all else equal, in a competitive environment. Since its state price $\Lambda_{j}(z)$ is more exposed to its own output $y_{j}(z) k_{j}$ because of diminished risk-sharing opportunities $\left(\Lambda_{j}(z)\right.$ lower when $y_{i}(z)$ is higher, and vice versa), it follows from the FONC for capital that agent $j$ chooses a lower level of capital $k_{j}$ than it would in a competitive environment. By symmetric arguments, all agents that produce in a competitive environment choose a lower level of capital as a result of market power.

The above, however, may not hold for agents that do not produce in a competitive equilibrium, as inferior production technologies may be utilized. Consider the extreme case where agent $i$ only lends in a competitive equilibrium but is effectively pushed into autarky with market power. Agent $i$ will then invest in capital if $\sum_{z \in \mathcal{Z}} y_{i}(z)>R_{f}$. Consequently, we expect all agents that do not produce in a competitive environment to choose a (weakly) higher level of capital as a result of market power.

To see that market power and limited commitment are substitutive effects, we recognize that both market power and limited commitment lead to an underprovision of state contingent securities. Since market power shades down how many state contingent claims agent $i$ issues on state $z$, and therefore borrows less against state $z$, it is less likely that $i^{\prime} s$ borrowing constraint will bind.

For the second claim, suppose $i$ indirectly intermediates funds to agent $j$. Comparing the expressions for the price of a state contingent claim referencing state $z$ issued by agent $j$ from Proposition 5, we see that if agent $i$ both issues claims $\hat{a}_{i}(i, z)$ and buys claims $\hat{a}_{i}(j, z)$ 
from agent $j$, then it must be the case that:

$$
q_{i}(z)-q_{j}(z)=-h_{i} \frac{\partial q_{i}(z)}{\partial \hat{a}_{i}(i, z)} \hat{a}_{i}(i, z)+h_{i} \frac{\partial q_{j}(z)}{\partial \hat{a}_{i}(j, z)} \hat{a}_{i}(j, z)>0 .
$$

Agent $i$ earns an intermediation spread $-h_{i} \frac{\partial q_{i}(z)}{\partial a_{i}(i, z)} \hat{a}_{i}(i, z)+h_{i} \frac{\partial q_{j}(z)}{\partial a_{i}(j, z)} \hat{a}_{i}(j, z)$ that acts as a wedge between the price at which it sells claims and that at which it buys claims to the same state, and this is true regardless of whether agent $i^{\prime} s$ constraint on $\hat{a}_{i}(i, z)$ binds. Even though the claims $\hat{a}_{i}(i, z)$ and $\hat{a}_{i}(j, z)$ net, it is their sum and not their difference that determines market power since agent $i$ is trading in segmented markets.

Notice now that the intermediation spread is increasing in both how many claims agent $i$ issues, $\hat{a}_{i}(i, z)$, and how much it buys, $\hat{a}_{i}(j, z)$. Furthermore, buying more claims $\hat{a}_{i}(j, z)$ has two effects: 1$)$ since these obligations net at $t=2$, such that agent $i$ receives $\hat{a}_{i}(j, z)+\hat{a}_{i}(i, z)$ in net transfers in state $z$; and 2) buying more claims $\hat{a}_{i}(j, z)$ relaxes the I.C. constraint of agent $i$, allowing it to issue $\xi \hat{a}_{i}(j, z)$ more claims to its creditors.

We can further examine the profit to exploiting this trading opportunity. Notice that, by buying $\hat{a}_{i}(j, z)$ and issuing $\hat{a}_{i}(i, z)$, the agent owes a net cash flow of $-\hat{a}_{i}(i, z)-\hat{a}_{i}(j, z)$ at $t=2$, and receives $-q_{i}(z) \hat{a}_{i}(i, z)-q_{j}(z) \hat{a}_{i}(j, z)$ at $t=1$. Consequently, the excess profit to agent $i, \Pi_{i j}(z)$, per dollar of equity is:

$$
\begin{aligned}
\Pi_{i j}(z)= & -r_{i} q_{i}(z) \hat{a}_{i}(i, z)-r_{i} q_{j}(z) \hat{a}_{i}(j, z)+\hat{a}_{i}(j, z)+\hat{a}_{i}(i, z) \\
= & -r_{i}\left(-h_{i} \frac{\partial q_{i}(z)}{\partial \hat{a}_{i}(i, z)} \hat{a}_{i}(i, z)+h_{i} \frac{\partial q_{j}(z)}{\partial \hat{a}_{i}(j, z)} \hat{a}_{i}(j, z)\right) \hat{a}_{i}(i, z)+\left(r_{i} q_{j}(z)-1\right)\left(\hat{d}_{i}(z)-\hat{a}_{i}(j, z)\right) \\
= & r_{i} h_{i}\left(\frac{\partial q_{i}(z)}{\partial \hat{a}_{i}(i, z)} \hat{a}_{i}(i, z)^{2}+\frac{\partial q_{j}(z)}{\partial \hat{a}_{i}(j, z)} \hat{a}_{i}(j, z)^{2}\right) \\
& +\left(1-\pi(z) \frac{u_{2}^{\prime}\left(\hat{c}_{i 2}(z)\right)+\xi \psi_{i}(z)}{E\left[u_{2}^{\prime}\left(\hat{c}_{i 2}(z)\right)+\xi \psi_{i}(z)\right]}\right)\left(\hat{a}_{i}(j, z)+\hat{a}_{i}(i, z)\right)
\end{aligned}
$$

where $r_{i}=\frac{1}{\sum_{z \in \mathcal{Z}} \Lambda_{i}(z)}$ is the effective risk-free rate for agent $i$, and we have substituted for $q_{j}(z)$ with the FONC from Proposition 5. Adding across agents $j$, and recognizing that $\frac{\pi(z)\left(u_{2}^{\prime}\left(\hat{c}_{i 2}(z)\right)+\xi \psi_{i}(z)\right)}{E\left[u_{2}^{\prime}\left(\hat{c}_{i 2}(z)\right)+\xi \psi_{i}(z)\right]}=\frac{\Lambda_{i}(z)}{\sum_{z \in \mathcal{Z}} \Lambda_{i}(z)}$, we arrive at the expression in the statement of the proposition.

Since the second term in parentheses is nonnegative, the second term is positive whenever $\sum_{j=1}^{N} \hat{a}_{i}(j, z) \geq 0$. Agent $i$ therefore earns a positive profit from being a net lender in state $z$ and, since $\frac{x}{a+x}$ is increasing in $x$, this profit is smaller if the debt constraint binds in state $z$. 
The first term is a profit (strict arbitrage) from agent $i^{\prime} s$ market power. This term is increasing in lending activity when $\frac{\partial q_{j}(z)}{\partial \hat{a}_{j}(i, z)}$ is an increasing function, which is satisfied when $f_{i}(\cdot, \cdot)$ is a convex function of its first argument. To see this, we recognize that, letting $x_{j}=-\sum_{i=1}^{N} h_{i} \hat{a}_{i}(j, z):$

$$
\frac{\partial^{2} q_{j}(z)}{\partial \hat{a}_{i}(j, z)^{2}}=\frac{\partial}{\partial \hat{a}_{i}(j, z)}\left(\frac{\partial f_{j}\left(x_{j}, z\right)^{-1}}{\partial x_{j}} \frac{\partial x_{j}}{\partial a_{i}(j, z)}\right)=-\frac{\partial x_{j}}{\partial a_{i}(j, z)} \frac{\partial f_{j}\left(x_{j}, z\right)^{-2}}{\partial x_{j}} \frac{\partial^{2} f_{j}\left(x_{j}, z\right)}{\partial x_{j}^{2}}
$$

from which follows that $\frac{\partial^{2} q_{j}(z)}{\partial a_{i}(j, z)^{2}} \geq 0$ whenever $\frac{\partial^{2} f_{j}\left(x_{j}, z\right)}{\partial x_{j}^{2}} \geq 0 \forall(j, z)$, and similarly:

$$
\frac{\partial^{2} q_{j}(z)}{\partial \hat{a}_{j}(j, z)^{2}}=-\frac{\partial x_{i}}{\partial \hat{a}_{j}(j, z)} \frac{\partial f_{i}\left(x_{i}, z\right)^{-2}}{\partial x_{i}} \frac{\partial^{2} f_{i}\left(x_{i}, z\right)}{\partial x_{i}^{2}} \geq 0
$$

whenever $\frac{\partial^{2} f_{i}\left(x_{i}, z\right)}{\partial x_{i}^{2}} \geq 0 \forall(i, z){ }^{21}$ Since $q_{i}(z)=f_{i}^{-1}(\cdot, z)$, and similarly with $q_{j}(z)$, this requirement is equivalent to requiring that $q_{i}(z)$ and $q_{j}(z)$ are convex in net demand.

Proof of Proposition 5:

Consider agents in the network. Let the expected excess payoff to the average statecontingent claim in state $z$ be $R(z)=\pi(z)-r^{*} E^{*}\left[q_{j}(z)\right]$, where $\frac{1}{r^{*}}=E\left[E^{*}\left[\Lambda_{i}(z)\right]\right]=$ $E\left[E^{*}\left[\frac{u_{2}^{\prime}\left(\hat{c}_{i 2}(z)\right)+\xi \psi_{i}(z)}{u_{1}^{\prime}\left(\hat{c}_{i 1}\right)}\right]\right]$ and $E^{*}[\cdot]$ is the cross-sectional average $\frac{1}{|N|} \sum_{i=1}^{|N|}[\cdot] \cdot r^{*}$ is the stateprice implied risk-free rate constructed from the cross-sectional average of agent state prices. Then, it follows that we can express this return from the FONCs for $\hat{b}_{i j}(z)$ and $\hat{d}_{i}(z)$ as:

$$
\begin{aligned}
R(z) & =\pi(z)-r^{*} \pi(z) E^{*}\left[\frac{u_{2}^{\prime}\left(\hat{c}_{i 2}(z)\right)+\xi \psi_{i}(z)}{u_{1}^{\prime}\left(\hat{c}_{i 1}\right)}\right]+r^{*} E^{*}\left[\frac{\partial q_{i}(z)}{\partial \hat{a}_{i}(i, z)} \hat{a}_{i}(i, z)\right] \\
& =-\operatorname{Cov}\left(\frac{E^{*}\left[\frac{u_{2}^{\prime}\left(\hat{c}_{i 2}(z)\right)+\xi \psi_{i}(z)}{u_{1}^{\prime}\left(\hat{c}_{i 1}\right)}\right]}{E\left[E^{*}\left[\frac{u_{2}^{\prime}\left(\hat{c}_{i 2}(z)\right)+\xi \psi_{i}(z)}{u_{1}^{\prime}\left(\hat{c}_{i 1}\right)}\right]\right]}, \delta(z)\right)+r^{*} E^{*}\left[h_{i} \frac{\partial q_{i}(z)}{\partial \hat{a}_{i}(i, z)} \hat{a}_{i}(i, z)\right],
\end{aligned}
$$

since $E\left[\frac{E^{*}\left[\Lambda_{i}(z)\right]}{\sum_{z \in \mathcal{Z}} E^{*}\left[\Lambda_{i}(z)\right]}\right] E[\delta(z)]=\pi(z)$ and $E\left[\frac{E^{*}\left[\Lambda_{i}(z)\right]}{\sum_{z \in \mathcal{Z}} E^{*}\left[\Lambda_{i}(z)\right]} \delta(z)\right]=\frac{E^{*}\left[\Lambda_{i}(z)\right]}{\sum_{z \in \mathcal{Z}} E^{*}\left[\Lambda_{i}(z)\right]} \pi(z)$, with

\footnotetext{
${ }^{21}$ Essentially, we require $f_{i}^{-1}(x, z)$ to be concave in its first argument $\forall(i, z)$. This is the case if $f_{i}(x, z)$ is convex, since $f_{i}^{-1}(x, z)$ is the inverse of $f_{i}(x, z)$.
} 
the convention that $\hat{b}_{j j}(z)=\hat{d}_{j}(z)$. We can further decompose this average excess payoff as:

$$
\begin{aligned}
R(z)= & -\operatorname{Cov}\left(\frac{E^{*}\left[\frac{u_{2}^{\prime}\left(\hat{c}_{i 2}(z)\right)}{u_{1}^{\prime}\left(\hat{c}_{i 1}\right)}\right]}{E\left[E^{*}\left[\frac{u_{2}^{\prime}\left(\hat{c}_{i 2}(z)\right)}{u_{1}^{\prime}\left(\hat{c}_{i 1}\right)}\right]\right]}, \delta(z)\right)-\operatorname{Cov}\left(\frac{E^{*}\left[\xi \psi_{i}(z)\right]-E^{*}\left[\frac{u_{2}^{\prime}\left(\hat{c}_{i 2}(z)\right)}{u_{1}^{\prime}\left(\hat{c}_{i 1}\right)}\right] \frac{E\left[E^{*}\left[\xi \psi_{i}(z)\right]\right]}{E\left[E^{*}\left[\frac{u_{2}^{\prime}\left(\hat{c}_{i 2}(z)\right)}{u_{1}^{\prime}\left(\hat{c}_{i 1}\right)}\right]\right]}}{E\left[E^{*}\left[\frac{u_{2}^{\prime}\left(\hat{c}_{i 2}(z)\right)+\xi \psi_{i}(z)}{u_{1}^{\prime}\left(\hat{c}_{i 1}\right)}\right]\right]}, \delta(z)\right. \\
& -r^{*} E^{*}\left[h_{i} \frac{\partial q_{i}(z)}{\partial \hat{a}_{i}(i, z)}\right]
\end{aligned}
$$

which we can rewrite as:

$$
R(z)=-\operatorname{Cov}\left(\bar{u}_{2}^{\prime}(z), \delta(z)\right)+\overline{m k t}(z)-\operatorname{Cov}(\overline{\operatorname{Lev}}(z), \delta(z)),
$$

where $\overline{m k t}(z)=r^{*} E^{*}\left[h_{i} \frac{\partial q_{i}(z)}{\partial \hat{a}_{i}(i, z)} \hat{a}_{i}(i, z)\right] \leq 0$, since $\frac{\partial q_{i}(z)}{\partial \hat{a}_{i}(i, z)} \geq 0, \bar{u}^{\prime}(z)=\frac{E^{*}\left[\frac{u_{2}^{\prime}\left(\hat{c}_{i 2}(z)\right)}{u_{1}^{\prime}\left(\hat{c}_{i 1}\right)}\right]}{E\left[E^{*}\left[\frac{u_{2}^{\prime}\left(\hat{c}_{i 2}(z)\right)}{u_{1}^{\prime}\left(\hat{c}_{i 1}\right)}\right]\right]}$, and $\overline{\operatorname{Lev}}(z)=\frac{E^{*}\left[\xi \psi_{i}(z)\right]-E^{*}\left[\frac{u_{2}^{\prime}\left(\hat{c}_{i 2}(z)\right)}{u_{12}^{\prime}\left(\hat{c}_{i 1}\right)}\right] \frac{E\left[E^{*}\left[\xi \psi_{i}(z)\right]\right]}{E\left[E^{*}\left[\frac{u_{2}^{\prime}\left(\hat{c}_{i 2}(z)\right)}{u_{1}^{\prime}\left(\hat{c}_{i 1}\right)}\right]\right]}}{E\left[E^{*}\left[\frac{u_{2}^{\prime}\left(\hat{c}_{i 2}(z)\right)+\xi \psi_{i}(z)}{u_{1}^{\prime}\left(\hat{c}_{i 1}\right)}\right]\right]}$.

For the second claim, we first recognize from the FOCs in the trading game that, if there is storage, then $E\left[u_{2}^{\prime}\left(\frac{c_{i 2}(z)}{c_{i 1}}\right)\right]=\frac{1}{R_{f}}$, and, if there is no storage, then $E\left[u_{2}^{\prime}\left(\frac{c_{i 2}(z)}{c_{i 1}}\right)\right]<\frac{1}{R_{f}}$. Consequently, if all agents invest only in storage, then the state-price implied risk-free rate, $r^{*}$, is $R_{f}$. To see that this is a lower bound, a necessariy condition that agent $i$ to invest in capital in its production technology is $E\left[y_{i}(z)\right]>R_{f}$, with the strict inequality necessary to embed a risk premium for the agent. Consequently, as long as agent $i$ holds storage, then $E\left[u_{2}^{\prime}\left(\frac{c_{i 2}(z)}{c_{i 1}}\right)\right]=\frac{1}{R_{f}}$, and only once it exhausts all its resources in state contingent claims and capital, then $E\left[u_{2}^{\prime}\left(\frac{c_{i 2}(z)}{c_{i 1}}\right)\right]<\frac{1}{R_{f}}$. Since this holds for all agents, it follows that $r^{*} \geq R_{f}{ }^{22}$

We next recognize that, in the first-best equilibrium without leverage constraints or market power, all agents consume fixed fractions of the aggregate resources each period, $\tilde{c}_{1, a g g}$ and $\tilde{c}_{2, a g g}(z)$, and their marginal utilities are aligned state-by-state $\left(E^{*}\left[\frac{\hat{c}_{i 2}^{F B}(z)}{\hat{c}_{i 1}^{F B}}\right]=\right.$ $\left.\frac{\tilde{c}_{2, a g g}(z)}{\tilde{c}_{1, a g g}} \forall i\right)$. This is confirmed in Proposition 6 .

As an intermediate step, we next compare the first-best economy to a pseudo-economy that has the same capital and storage choices as in the decentralized economy, viewed now as agent endowments, but with perfect risk-sharing. In this pseudo-economy, all agents

\footnotetext{
${ }^{22}$ Though agents will not trade risk-free claims with each other because of market power, they may still trade with outside investors as a result of their difference in preferences.
} 
consume a fixed fraction of their aggregate output each period, $c_{1, a g g}^{*}$ and $c_{z, a g g}^{*}(z)$, but this endowment reflects the capital and savings decisions of the true noncompetitive economy.

From Proposition 4 and Corollary 4, agents that would invest in capital in the first-best underinvest in the noncompetitive economy because of strategic frictions, while those that do not invest in capital may start to invest because of diminished opportunities to invest. For the same resources transferred intertemporally, $\sum_{i=0}^{N} e_{i}-\tilde{c}_{1, a g g}$, the first-best employs more efficient technologies without cross-sectional misallocation. The first-best optimally increases the average level of consumption in all states, and shifs it from less probable to more likely states of the world, lowering marginal utility in those states and the average marginal utility, and consequently raising the risk-free rate. Since the decentralized production frontier is in the interior of the first-best production possibilities set, it is less efficient in shifting consumption across states of the world, leading to higher average marginal utility, and a lower riskless rate.

What remains for us to establish now is that the risk-free rate in the noncompetitive economy is (weakly) lower than in the psuedo-economy with perfect risk-sharing, since then the result follows by transitivity. We now recognize that:

$$
\sum_{z \in \mathcal{Z}} E^{*}\left[\Lambda_{i}(z)\right] \geq E\left[E^{*}\left[u_{2}^{\prime}\left(\frac{c_{i 2}(z)}{c_{i 1}}\right)\right]\right]
$$

where the inequality, similar to Alvarez and Jermann (2000), follows from the possibility of binding leverage constraints and the homotheticity of agent utility. As a result of market power, these inequalities can hold strictly even when no leverage constraints bind. Since $\sum_{i=1}^{|N|} c_{i 2}(z)<c_{z, \text { agg }}^{*}(z)$ and $\sum_{i=1}^{|N|} c_{i 1}=c_{1, a g g}^{*}, \frac{E^{*}\left[c_{i 2}(z)\right]}{E^{*}\left[c_{i 1}\right]}<\frac{c_{z, a g g}^{*}(z)}{c_{1, a g g}^{*}} \forall z \in \mathcal{Z}$, and therefore $E\left[u_{2}^{\prime}\left(\frac{E^{*}\left[c_{i 2}(z)\right]}{E^{*}\left[c_{i 1}\right]}\right)\right]>E\left[u_{2}^{\prime}\left(\frac{c_{z, a g g}^{*}(z)}{c_{1, a g g}^{*}}\right)\right]$ since marginal utility is convex. It then follows that it is sufficient that:

$$
E\left[E^{*} u_{2}^{\prime}\left(\frac{c_{i 2}(z)}{c_{i 1}}\right)\right] \geq E\left[u_{2}^{\prime}\left(\frac{E^{*}\left[c_{i 2}(z)\right]}{E^{*}\left[c_{i 1}\right]}\right)\right]
$$

for the state-price implied riskless rate rate, $r^{*}$, to be less than that in the first-best economy.

Finally, for the third claim, notice that $R(z)$ is the expected excess payoff in state $z$. Summing across all states, it follows that:

$$
\sum_{z \in \mathcal{Z}} R(z)=\sum_{z \in \mathcal{Z}} \overline{m k t}(z) \leq 0
$$


since $\sum_{z \in \mathcal{Z}} \operatorname{Cov}\left(\bar{u}_{2}^{\prime}(z)+\overline{\operatorname{Lev}}(z), \delta_{j}(z)\right)=0$. Consequently, $\sum_{z \in \mathcal{Z}} R(z)$ is nonzero despite being a riskless expected excess return derived from assembling all state contingent securities. It must therefore be the case that the market-implied riskless rate $\left[\sum_{z \in \mathcal{Z}} E^{*}\left[q_{j}(z)\right]\right]^{-1}$ is biased downward based on the average market power, $\sum_{z \in \mathcal{Z}} \overline{m k t}(z)$, in financial markets.

Proof of Proposition 6 :

We first characterize the first-best allocation. Since all agent links allow for both the sale and purchase of state contingent claims, resources can potentially flow to any agent.

Without constraints on allocations, the Social Planner solves the problem:

$$
\begin{aligned}
& \sup _{\left\{\vec{c}_{1}(z), \vec{k}\right\}} \sum_{i=1}^{|N|} u_{2}^{\prime}\left(1 / \eta_{i}\right) u_{1}\left(c_{i 1}\right)+\sum_{z \in \mathcal{Z}} \pi(z) u_{2}^{\prime}\left(1 / \eta_{i}\right) u_{2}\left(c_{i 2}(z)\right)+\sum_{z \in \mathcal{Z}} \pi(z) u_{2}^{\prime}\left(1 / \eta_{0}\right) F\left(c_{02}(z)\right), \\
\text { s.t. }: & \sum_{i=0}^{|N|}\left(c_{i 2}(z)-y_{i}(z) k_{i}-R_{f} s_{i}\right) \leq 0 \forall z \in \mathcal{Z}, \\
: & \sum_{i=1}^{|N|} k_{i}+c_{i 1}+s_{i}-e_{i}-e_{0} \leq 0
\end{aligned}
$$

where $u_{2}^{\prime}\left(1 / \eta_{i}\right)$ are the Pareto weights and $s_{0}=k_{0}=0$. In the above, we have implicitly given equal Pareto weights to the cross-section of outside investors by issuer with a state $z$.

With Lagrange multipliers on the feasibility conditions $\lambda$ and $\mu(z) \pi(z)$, it follows that the FOC for $c_{i 1}, c_{i 2}(z)$, and $k_{i}$ are:

$$
\begin{aligned}
c_{i 1}: & u_{2}^{\prime}\left(1 / \eta_{i}\right) u_{1}^{\prime}\left(c_{i 1}\right)-\lambda \leq 0,\left(=\text { if } c_{i 1}>0\right), \\
c_{i 2}(z): & u_{2}^{\prime}\left(1 / \eta_{i}\right) u_{2}^{\prime}\left(c_{i 2}(z)\right)-\mu(z) \leq 0,\left(=\text { if } c_{i 2}(z)>0\right), \\
c_{02}(z): & u_{2}^{\prime}\left(1 / \eta_{0}\right) F^{\prime}\left(c_{02}(z)\right)-\mu(z) \leq 0,\left(=\text { if } c_{i 0}(z)>0\right) \\
k_{i}: & \sum_{z \in \mathcal{Z}} \pi(z) \mu(z) y_{i}(z)-\lambda \leq 0\left(=\text { if } k_{i}>0\right), \\
s_{i} & : \quad R_{f} \sum_{z \in \mathcal{Z}} \pi(z) \mu(z)-\lambda \leq 0\left(=\text { if } s_{i}>0\right),
\end{aligned}
$$

Since the utility function satisfies the Inada condition, in equilibrium $c_{i 1}, c_{i 2}(z)>0$.

Let $c_{i 1}=\hat{c}_{i 1} \sum_{i=0}^{|N|} e_{i}, c_{i 2}(z)=\hat{c}_{i 2}(z) \sum_{i=0}^{|N|} e_{i}$ and $k_{i}=\hat{k}_{i} \sum_{i=0}^{|N|} e_{i}$. It is straightforward to see from the FOC for consumption, the homotethicity of the utility functions, and the 
feasibility constraint that:

$$
\begin{aligned}
c_{i 1} & =\frac{\eta_{i}}{\sum_{i=1}^{N} \eta_{i}} \sum_{i=1}^{|N|} c_{i}=\frac{\eta_{i}}{\sum_{i=1}^{N} \eta_{i}} \tilde{c}_{1, a g g}, \\
c_{02}(z) & =F^{-1 \prime} \cdot u_{2}^{\prime}\left(\frac{\eta_{i}}{\sum_{i=0}^{N} \eta_{i}} \tilde{c}_{2, a g g}(z)\right) \\
c_{i 2}(z) & =\frac{\eta_{i}}{\sum_{i=0}^{N} \eta_{i}} \tilde{c}_{2, a g g}(z), i \in\{1, \ldots, N\},
\end{aligned}
$$

where:

$$
\tilde{c}_{2, a g g}(z)=u_{2}^{\prime}\left(F\left(c_{02}(z)\right)\right)-c_{02}(z)+\sum_{i=1}^{|N|} R_{f}\left(1-c_{i 1}\right)+\left(y_{i}(z)-R_{f}\right) k_{i}
$$

is the aggregate effective resources in each state $z$. The first FOC implies that $\lambda=u_{1}^{\prime}\left(\tilde{c}_{1, a g g} / \sum_{i=1}^{N} \eta_{i}\right)$, while the second that $\mu(z)=u_{2}^{\prime}\left(\tilde{c}_{2, a g g}(z) / \sum_{i=0}^{N} \eta_{i}\right)$, from which follows that the fourth and fifth FOCs reduce to:

$$
\begin{gathered}
\sum_{z \in \mathcal{Z}} \pi(z) u_{2}^{\prime}\left(\frac{\sum_{i=1}^{N} \eta_{i}}{\sum_{i=0}^{N} \eta_{i}}\right) \frac{u_{2}^{\prime}\left(\tilde{c}_{2, a g g}(z)\right)}{u_{1}^{\prime}\left(\tilde{c}_{1, a g g}\right)} y_{i}(z) \leq 1\left(=\text { if } k_{i}>0\right), \\
R_{f} \sum_{z \in \mathcal{Z}} \pi(z) u_{2}^{\prime}\left(\frac{\sum_{i=1}^{N} \eta_{i}}{\sum_{i=0}^{N} \eta_{i}}\right) \frac{u_{2}^{\prime}\left(\tilde{c}_{2, a g g}(z)\right)}{u_{1}^{\prime}\left(\tilde{c}_{1, a g g}\right)} \leq 1(=\text { if } s>0),
\end{gathered}
$$

where we drop the subscript for storage since only the aggregate level of storage is determinate.

Suppos that production technology $y_{i}(z)$ is employed. Since the technology is employed, it must be the case that $\sum_{z \in \mathcal{Z}} \pi(z) u_{2}^{\prime}\left(c_{j 2}(z)\right) y_{i}(z) \geq R_{f} \sum_{z \in \mathcal{Z}} \pi(z) u_{2}^{\prime}\left(c_{j 2}(z)\right)$, and consequently:

$$
u_{1}^{\prime}\left(c_{j 1}\right)=\sum_{z \in \mathcal{Z}} \pi(z) u_{2}^{\prime}\left(c_{j 2}(z)\right) y_{i}(z) \geq R_{f} \sum_{z \in \mathcal{Z}} \pi(z) u_{2}^{\prime}\left(c_{j 2}(z)\right)
$$

and therefore consumption is a submartingale.

Notice that, if $k_{i}>0$, then it follows, since $\sum_{z \in \mathcal{Z}} \pi(z) u_{2}^{\prime}\left(\frac{\sum_{i=1}^{N} \eta_{i}}{\sum_{i=0}^{N} \eta_{i}}\right) \frac{u_{2}^{\prime}\left(\tilde{c}_{2, a g g}(z)\right)}{u_{1}^{\prime}\left(\tilde{c}_{1, a g g}\right)} \leq \frac{1}{R_{f}}$, that:

$$
\sum_{z \in \mathcal{Z}} \pi(z) y_{i}(z)>R_{f}
$$


since all production technologies $y_{i}(z)$ are (weakly) positively correlated, and therefore $\operatorname{Cov}\left(u_{2}^{\prime}\left(\tilde{c}_{2, a g g}(z)\right), y_{i}(z)\right)<0$.

Notice that, if $\vec{y}_{i} \geq \vec{y}_{j}$, with at least one inequality strict, then, if $k_{i}>0$, it must be the case that $k_{j}=0$, since:

$$
\sum_{z \in \mathcal{Z}} \pi(z) u_{2}^{\prime}\left(\frac{\sum_{i=1}^{N} \eta_{i}}{\sum_{i=0}^{N} \eta_{i}}\right) \frac{u_{2}^{\prime}\left(\tilde{c}_{2, a g g}(z)\right)}{u_{1}^{\prime}\left(\tilde{c}_{1, a g g}\right)}\left(y_{i}(z)-y_{j}(z)\right)<0
$$

given that $\frac{u_{2}^{\prime}\left(\tilde{c}_{2, a g g}(z)\right)}{u_{1}^{\prime}\left(\tilde{c}_{1, a g g}\right)} \geq 0$ and $\vec{y}_{i} \geq \vec{y}_{j}$, and therefore:

$1=\sum_{z \in \mathcal{Z}} \pi(z) u_{2}^{\prime}\left(\frac{\sum_{i=1}^{N} \eta_{i}}{\sum_{i=0}^{N} \eta_{i}}\right) \frac{u_{2}^{\prime}\left(\tilde{c}_{2, a g g}(z)\right)}{u_{1}^{\prime}\left(\tilde{c}_{1, a g g}\right)} y_{i}(z)>\sum_{z \in \mathcal{Z}} \pi(z) u_{2}^{\prime}\left(\frac{\sum_{i=1}^{N} \eta_{i}}{\sum_{i=0}^{N} \eta_{i}}\right) \frac{u_{2}^{\prime}\left(\tilde{c}_{2, a g g}(z)\right)}{u_{1}^{\prime}\left(\tilde{c}_{1, a g g}\right)} y_{j}(z)$.

We now demonstrate that this first-best allocation is infeasible in the decentralized economy because of market power. Rewriting FOCs with complementary slackness, summing over the states $z \in \mathcal{Z}$ for the first two FONCs, and substituting them into the budget constraint reveals that:

$\sum_{z \in \mathcal{Z}} \pi(z) \frac{u_{2}^{\prime}\left(c_{i 2}(z)\right)}{u_{1}^{\prime}\left(c_{i 1}\right)} c_{i 2}(z)=e_{i}-c_{i 1}+e_{i} \sum_{z \in \mathcal{Z}}\left(-\frac{\partial q_{i}(z)}{\partial a_{i}(i, z)} a_{i}(i, z)+\frac{\partial \vec{q}(z)^{\prime}}{\partial \overrightarrow{\hat{a}}_{-i}(z)} \overrightarrow{\hat{a}}_{-i}(z)\right) \geq e_{i}-c_{i 1}$

where the $\psi_{i}(z)$ terms cancel because of the complementary slackness condition on the I.C. constraint. The above condition implies that value of the wealth portfolio exceeds initial invested wealth $e_{i}-c_{i 1}$ because of market power, implying the agent earns a higher return because it internalizes its price impact.

If there is perfect-risk sharing, then the marginal utilities of all agents are aligned stateby-state, and there is a unique pricing kernel. Adding the equilibrium condition across agents implies that:

$\sum_{i=1}^{|N|} c_{i 1}+\sum_{z \in \mathcal{Z}} \Lambda(z) \sum_{i=1}^{|N|} c_{i 2}(z)=\sum_{i=1}^{|N|} e_{i}+\sum_{i=1}^{|N|} e_{i} \sum_{z \in \mathcal{Z}}\left(-\frac{\partial q_{i}(z)}{\partial a_{i}(i, z)} a_{i}(i, z)+\frac{\partial \vec{q}(z)^{\prime}}{\partial \overrightarrow{\hat{a}}_{-i}(z)} \overrightarrow{\hat{a}}_{-i}(z)\right)$

where $\Lambda(z)$ is the unique state price deflator. In contrast, for the outside investors:

$$
\frac{\pi(z) F^{\prime}\left(\frac{1}{N|\mathcal{Z}|} e_{0}+\left(1-r q_{i}(z)\right) D(i, z), z\right)}{\sum_{z \in \mathcal{Z}} \pi(z) F^{\prime}\left(\frac{1}{N|\mathcal{Z}|} e_{0}+\left(1-r q_{i}(z)\right) D(i, z), z\right)}=r q_{i}(z), \forall(i, z)
$$


which implies, by complete markets, that:

$$
\sum_{z \in \mathcal{Z}} \Lambda(z) c_{02}(z)=\frac{1}{R} e_{0}
$$

where $R=\frac{1}{\sum_{z \in \mathcal{Z}} \Lambda(z)}$ is the effective risk-free rate. Consequently, outside investors' portfolios are correctly priced.

It then follows, since $-\frac{\partial q_{i}(z)}{\partial a_{i}(i, z)} a_{i}(i, z)+\frac{\partial \vec{q}(z)^{\prime}}{\partial \overrightarrow{\hat{a}}_{-i}(z)} \overrightarrow{\hat{a}}_{-i}(z)$ is nonnegative for all $i$ and $z$, that:

$$
\sum_{i=1}^{|N|} c_{i 1}+\sum_{z \in \mathcal{Z}} \pi(z) c_{02}(z)+\sum_{z \in \mathcal{Z}} \Lambda(z) \sum_{i=1}^{|N|} c_{i 2}(z)>\frac{1}{R} e_{0}+\sum_{i=1}^{|N|} e_{i}
$$

and that the present discounted value of aggregate consumption exceeds the total value of all equity in the economy at $t=1$. This is a contradiction since it implies the aggregate wealth portfolio is mispriced, or that there is an aggregate economic profit from trading.

For the final part of the claim, we consider the perspective of a social planner who can design a mechanism to implement any allocation that respects the FOCs of all agents and the market structure that features imperfect competition. Expanding the differential of the indirect utility function for agent $i, U_{1}^{i}$, we see that:

$$
\begin{aligned}
\Delta U_{i}= & u_{1}^{\prime}\left(c_{i 1}\right) \Delta c_{i 1}+E\left[u_{2}^{\prime}\left(c_{i 2}\right) \Delta c_{i 2}\right]+\Delta\left(\sum_{z \in \mathcal{Z}} \pi(z) \psi_{i}(z)\left(a_{i}(i, z)+\xi y_{i}(z) k_{i}+\xi \vec{b}_{i}(z) \iota+\xi R_{f} s_{i}\right)\right) \\
& +\Delta\left(\phi_{i}^{k} k_{i}\right)+\Delta\left(\phi_{i}^{s} s_{i}\right)-\Delta\left(\phi_{i}^{a} a_{i}(i, z)\right)+\sum_{z \in \mathcal{Z}} \Delta\left(\vec{\phi}_{-i}^{a \prime} \vec{a}_{-i}(z)\right),
\end{aligned}
$$

where $\phi_{i}^{k}$ is the nonnegativity Lagrange multiplier on $k_{i}$, and similarly for the other $\phi_{i}^{\prime} s$. Substituting with the FOCs in Proposition 2, the above reduces to:

$$
\begin{aligned}
\Delta U_{i}= & u_{1}^{\prime}\left(c_{i 1}\right) \sum_{z \in \mathcal{Z}}\left(\left(-\Delta q_{i}(z)+\frac{\partial q_{i}(z)}{\partial a_{i}(i, z)} \Delta a_{i}(i, z)\right) a_{i}(i, z)\right) \\
& +u_{1}^{\prime}\left(c_{i 1}\right) \sum_{z \in \mathcal{Z}}\left(\sum_{j \neq i} a_{i}(j, z)\left(-\Delta q_{j}(z)+\frac{\partial q_{j}(z)}{\partial a_{i}(j, z)} \Delta a_{i}(j, z)\right)\right) \\
& +\sum_{z \in \mathcal{Z}} \pi(z) \Delta \psi_{i}(z)\left(a_{i}(i, z)+\xi y_{i}(z) k_{i}+\xi \vec{a}_{-i} \iota+\xi R_{f} s_{i}\right) \\
& +\Delta \phi_{i}^{k} k_{i}+\Delta \phi_{i}^{s} s_{i}-\Delta \phi_{i}^{a} a_{i}(i, z)+\sum_{z \in \mathcal{Z}} \Delta \vec{\phi}_{-i}^{a \prime} \vec{a}_{-i}(z)
\end{aligned}
$$


Finally, invoking complementary slackness and the definition of $q_{i}(z)$, we arrive at:

$$
\begin{aligned}
\Delta U_{i}= & -u_{1}^{\prime}\left(c_{i 1}\right) \sum_{z \in \mathcal{Z}} \Delta \Lambda_{i}(z)\left(a_{i}(i, z)+\vec{a}_{-i}(z) \iota\right) \\
& -u_{1}^{\prime}\left(c_{i 1}\right) \sum_{z \in \mathcal{Z}}\left(\sum_{j \neq i} \Delta\left(\frac{\partial q_{j}(z)}{\partial a_{i}(j, z)}\right) a_{i}(j, z)+\Delta\left(\frac{\partial q_{i}(z)}{\partial a_{i}(i, z)}\right) a_{i}(i, z)\right) .
\end{aligned}
$$

When markets are competitive, the second term vanishes, and $\Lambda_{i}(z)=\Lambda(z)$, since state contingent claim prices align state prices across agents. It then follows, since $a_{i}(i, z)+$ $\vec{a}_{-i}(z) \iota>0$ for some agent $i$ and $a_{j}(j, z)+\vec{a}_{-j}(z) \iota<0$ for some other agent $j$, generically, and $\Delta \Lambda_{i}(z)=\Delta \Lambda(z)$ is symmetric across agents, it follows that generically the trading game is constrained Pareto efficient. ${ }^{23}$

Autarky is an equilibrium in which $q(z)=\max _{i} \Lambda_{i}(z)$ to induce all agents to want to sell claims, which leads to excess supply in all markets. As a result, state prices of agents are not aligned state-by-state. If another competitive equilibrium exists, the Planner can improve upon the allocation by lowering asset prices sufficiently to induce trade. This would lower the marginal utility of buyers $\left(\Delta \Lambda_{i}(z) a_{i}(j, z)<0\right)$, and raise the marginal utility of sellers $\left(\Delta \Lambda_{i}(z) a_{i}(i, z)<0\right)$ as state prices are aligned, resulting in $\Delta U_{i}>0$ for now trading agents.

With imperfect competition, we now recognize that the second term reveals that a Planner would like to raise (lower) prices in securities for which agent $i$ borrows (lends). Since the pricing functions are symmetric across agents, $\frac{\partial q_{j}(z)}{\partial a_{i}(j, z)}=\frac{\partial q_{j}(z)}{\partial a_{j}(j, z)}$, it follows that manipulating prices is zero-sum, and would necessarily have to hurt the seller's (buyer's) utility at the expense of the buyer's (seller's). Consequently, the second term is compatible with constrained Pareto efficiency.

We focus now on the first term. As a result of market power, it is no longer true that $\Lambda_{i}(z)=\Lambda_{j}(z)$ across agents, since they intentionally under diversify risk to earn monopoly rents. Since $u_{2}^{\prime}\left(c_{i 1}\right)>0$, it follows that policies by a Planner that raise $\Lambda_{i}(z), \Delta \Lambda_{i}(z)>0$, when $i$ is a net borrower, $a_{i}(i, z)+\vec{a}_{-i}(z) \iota<0$, raises $i^{\prime} s$ utility by raising the price at which $i$ sells its securities, and similarly with $\Delta \Lambda_{i}(z)<0$ when $i$ is a net lender, $a_{i}(i, z)+\vec{a}_{-i}(z) \iota>0$. Since state prices are not aligned across agents, there can exist policies for which it is possible to improve $i^{\prime} s$ utility without harming that of other agents. Generically, then, the equilibrium

${ }^{23}$ This is a stronger statement than the Planner cannot improve utilitarian welfare, which also follows since summing across agents reveals that $\sum_{i} \Delta U_{i}=0$, when the outside agents are included in the calculation. 
in the trading game with imperfect competition is constrained Pareto inefficient.

Proof of Corollary 3 :

The claim follows from Proposition 6. Since the decentralized economies with limited commitment and/or market power cannot achieve the first-best allocation, the distribution of productivities across agents matters for investment and, consequently, asset prices. As such, any redistribution of productivities across agents state-by-state has a material impact on the production possibilities frontier, and therefore equilibrium outcomes, with the constrained efficient frontier being achieved with only limited commitment.

In contrast, with commitment and perfectly competitive agents, the competitive equilibrium attains the first-best allocation. It then follows from Proposition 6 that all equilibrium outcomes depend on only the most efficient production technologies.

\section{Proof of Corollary 4:}

Suppose all agents are competitive. Then, $q_{i}(z)=q(z) \forall i$ because agents are price takers, and the profit to agent $i$ from intermediating funds to $j$ in state $z$ is

$$
\begin{aligned}
\Pi_{i}(z) & =\left(1-\pi(z) \frac{u_{2}^{\prime}\left(\hat{c}_{i 2}(z)\right)+\xi \psi_{i}(z)}{E\left[u_{2}^{\prime}\left(\hat{c}_{i 2}(z)\right)+\xi \psi_{i}(z)\right]}\right)\left(\hat{a}_{i}(j, z)+\hat{a}_{i}(i, z)\right) \\
& =\left(1-\pi(z) \frac{u_{2}^{\prime}\left(\hat{c}_{i 2}(z)\right)+\xi \psi_{i}(z)}{E\left[u_{2}^{\prime}\left(\hat{c}_{i 2}(z)\right)+\xi \psi_{i}(z)\right]}\right) \tilde{a}_{i}(j, z),
\end{aligned}
$$

where $\tilde{a}_{i}(j, z)$ is the net debt agent $i$ buys. It then follows that the extent of indirect intermediation is irrelevant. Consequently, agent $i$ would earn the same profit from lending to $j$ without borrowing funds $\hat{a}_{i}(i, z)$, and incurring a cost $\frac{c}{2}$ for forming the additional link with its lender. As such, no agent would indirectly intermediate funds in the network.

Furthermore, since the identity of $i^{\prime} s$ counterparties no longer impacts its optimization in the trading game, as state-contingent claims prices are equated within the network, there are no network effects beyond price externalities. There are still network linkages since subsets of agents may not be able to trade with each other.

Finally, consider the FONC for capital for agent $i$ :

$$
\sum_{z \in \mathcal{Z}} \pi(z) \frac{u_{2}^{\prime}\left(c_{i 2}(z)\right)+\xi e_{i}^{-\gamma} \psi_{i}(z)}{u_{1}^{\prime}\left(c_{i 1}\right)} y_{i}(z) \leq 1 \quad\left(=\text { if } k_{i}>0\right)
$$


If capital were not collateralizable $\left(\pi(z) \frac{\xi e_{i}^{-\gamma} \psi_{i}(z)}{u_{1}^{\prime}\left(c_{i 1}\right)}\right.$ of the state price omitted), then when the borrowing constraint binds, agent $i$ is limited in its ability to sell claims to states in which it has a lot of resources, while it is always limited in its ability to buy claims to states in which it has few resources. As a result, agent $i$ is more exposed to its own production, reducing its optimal choice of capital. Since capital is collateralizable, however, having capital is also valuable because it relaxes the borrowing constraint across all states. Given that agent $i$ must be constrained for this offsetting value to capital to be in effect, it follows agent $i$ must still face rationed risk-sharing at its optimal choice of capital.

Consequently, agent $i$ that produces in the competitive equilibrium chooses a lower level of capital than in the absence of the borrowing constraint. Similar to the case of market power, we also expect all agents that do not produce in a competitive environment to choose a (weakly) higher level of capital as a result of limited commitment. 


\section{Appendix B: Examples of Outside Investor Demand}

From the main text, the optimization program of outside investors when choosing their demand for claims issued by agent $i$ in state $z$ satisfies the FONC:

$$
\frac{\pi(z)}{1-\pi(z)} \frac{F^{\prime}\left(r \frac{e_{0}}{N|\mathcal{Z}|}+\left(1-r q_{i}(z)\right) D(i, z), z\right)}{F^{\prime}\left(r \frac{e_{0}}{N|\mathcal{Z}|}-r q_{i}(z) D(i, z), z\right)} \frac{1-r q_{i}(z)}{r q_{i}(z)}=1 .
$$

Mean-Variance: In the case that outside investors have MV utility with coefficient of risk aversion $\lambda / \sum_{j=1}^{N} e_{j}$, it follows from their optimization program that:

$$
D(i, z)=\frac{\pi(z)-r q_{i}(z)}{\lambda \pi(z)(1-\pi(z))} \sum_{j=1}^{N} e_{j}
$$

and $f\left(q_{i}(z), z\right)=\frac{\pi(z)-r q_{i}(z)}{\lambda \pi(z)(1-\pi(z))}$. This gives rise to the linear pricing function:

$$
q_{i}(z)=\frac{1}{r} \pi(z)-\frac{\lambda}{r} \pi(z)(1-\pi(z)) \frac{-\sum_{j=1}^{N} a_{j}(i, z)}{\sum_{j=1}^{N} e_{j}},
$$

where $\tilde{\lambda}=\frac{\lambda}{r} \frac{\pi(z)(1-\pi(z))}{\sum_{j=1}^{N} e_{j}}$ has the interpretation of Kyle (1985)'s Lambda for purchasing agents, which is constant in this setting.

CARA Utility: In the case that outside investors have CARA utility with risk aversion $A / \sum_{j=1}^{N} e_{j}$, which scales inversely with total agent equity, then their demand is given by:

$$
D(i, z)=\left(\frac{1}{A} \log \frac{\pi(z)}{1-\pi(z)}+\frac{1}{A} \log \left(\frac{1}{r q_{i}(z)}-1\right)\right) \sum_{j=1}^{N} e_{j}
$$

and $f\left(q_{i}(z), z\right)=\frac{1}{\gamma} \log \frac{\pi(z)}{1-\pi(z)}+\frac{1}{\gamma} \log \left(\frac{1}{r q_{i}(z)}-1\right)$. This gives rise to the pricing function:

$$
q_{i}(z)=\frac{1}{r} \frac{1}{1+\frac{1-\pi(z)}{\pi(z)} \exp \left(A \frac{-\sum_{j=1}^{N} a_{j}(i, z)}{\sum_{j=1}^{N} e_{j}}\right)},
$$

which is quasilinear in net supply $-\sum_{j=1}^{N} a_{j}(i, z)$ (convex for $\sum_{j=1}^{N} a_{j}(i, z)<0$ and concave for $\left.\sum_{j=1}^{N} a_{j}(i, z) \geq 0\right)$.

CRRA Utility: In the case that outside investors instead have CRRA utility with coefficient of relative risk aversion $\gamma_{0}$, and assuming that $e_{0}=\frac{1}{N|\mathcal{Z}|} \sum_{j=1}^{N} e_{j}$, it follows their 
demand is given by:

$$
D(i, z)=\frac{r}{r q_{i}(z)+\frac{1}{\left(\frac{\pi(z)}{1-\pi(z)} \frac{1-r q_{i}(z)}{r q_{i}(z)}\right)^{\frac{1}{\gamma}}-1}} \frac{1}{N|\mathcal{Z}|} \sum_{j=1}^{N} e_{j}
$$

and $f\left(q_{i}(z), z\right)=\left(r q_{i}(z)+\frac{1}{\left(\frac{\pi(z)}{1-\pi(z)} \frac{1-r q_{i}(z)}{r q_{i}(z)}\right)^{\frac{1}{\gamma}}-1}\right)^{-1} r$. In the special case of log utility, then:

$$
D(i, z)=\frac{r}{1-r q_{i}(z)}\left(\frac{\pi(z)}{r q_{i}(z)}-1\right) \frac{1}{N|\mathcal{Z}|} \sum_{j=1}^{N} e_{j}
$$

which gives rise to the pricing function:

$q_{i}(z)=\left\{\begin{array}{l}\frac{1}{2 r}\left(1+\frac{\frac{r}{N|\mathcal{Z}|} \sum_{j=1}^{N} e_{j}}{-\sum_{j=1}^{N} a_{j}(i, z)}\right)+\frac{1}{2 r} \sqrt{\left(1+\frac{\frac{r}{N|\mathcal{Z}|} \sum_{j=1}^{N} e_{j}}{-\sum_{j=1}^{N} a_{j}(i, z)}\right)^{2}-\frac{4 \pi(z) \frac{r}{N|\mathcal{Z}|} \sum_{j=1}^{N} e_{j}}{-\sum_{j=1}^{N} a_{j}(i, z)}}, \sum_{j=1}^{N} a_{j}(i, z) \geq 0 \\ \left.\frac{1}{2 r}\left(1+\frac{r}{-\sum_{j=1}^{N} \sum_{j=1}^{N} e_{j}(i, z)}\right)-\frac{1}{2 r} \sqrt{\left(1+\frac{r}{N|\mathcal{Z}| \sum_{j=1}^{N} e_{j}}-\sum_{j=1}^{N} a_{j}(i, z)\right.}\right)^{2}-\frac{4 \pi(z) \frac{r}{N|\mathcal{Z}|} \sum_{j=1}^{N} e_{j}}{-\sum_{j=1}^{N} a_{j}(i, z)}, \sum_{j=1}^{N} a_{j}(i, z)<0\end{array}\right.$,

where we have selected the appropriate root of the quadratic form by imposing that $\pi(z) \rightarrow$ $0 \Longrightarrow q_{i}(z) \rightarrow 0^{+}$and $\pi(z) \rightarrow 1 \Longrightarrow q_{i}(z) \rightarrow \frac{1}{r}$. 